\title{
A Comparison Study of Copula Models for European Financial Index Returns
}

\author{
Paula V. Tófoli ${ }^{1}$, Flavio A. Ziegelmann ${ }^{2} \&$ Osvaldo Candido $^{1}$ \\ ${ }^{1}$ Graduate Program in Economics, Catholic University of Brasilia, Brasilia, Brazil \\ ${ }^{2}$ Graduate Program in Economics, Graduate Program in Management and Department of Statistics, Federal \\ University of Rio Grande do Sul, Porto Alegre, Brazil \\ Correspondence: Paula V. Tófoli, Graduate Program in Economics, Catholic University of Brasilia, Brasilia (DF), \\ SGAN 916, Module B, room A-120 - Asa Norte, 70790-160, Brazil. Tel: 55-61-3448-7192. E-mail: \\ paula.tofoli@ucb.br
}

Received: July 14, 2017

Accepted: September 5, 2017

Online Published: September 10, 2017

doi:10.5539/ijef.v9n10p155

URL: https://doi.org/10.5539/ijef.v9n10p155

\begin{abstract}
In this paper, we introduce a new approach to modeling dependence between international financial returns over time, combining time-varying copulas and the Markov switching model. We apply these copula models and also those proposed by Patton (2006), Jondeau and Rockinger (2006) and Silva Filho, Ziegelmann, and Dueker (2012) to the return data of the FTSE-100, CAC-40 and DAX indexes. We are particularly interested in comparing these methodologies in terms of the resulting dynamics of dependence and the models' abilities to forecast possible capital losses. Because risks related to extreme events are important for risk management, we compare and select the models based on VaR forecasts. Interestingly, all the models identify a long period of high dependence between the returns beginning in 2007, when the subprime crisis was evolving. Surprisingly, the elliptical copulas perform best in forecasting the extreme quantiles of the portfolios returns.
\end{abstract}

Keywords: copula-GARCH, IFM method, Markov switching model, time-varying copulas, value at risk

\section{Introduction}

In portfolio theory, multivariate financial returns are often assumed to be normally distributed, but, in practice, this assumption does not hold (Sancetta \& Satchell, 2001). Financial returns are found to be leptokurtic and they also show skewness in univariate distributions. Additionally, a number of studies have also reported asymmetry in the dependence amongst international financial returns, in the sense that they exhibit greater dependence during market downturns than during market upturns (see, for example, Longin \& Solnik, 2001, Ang \& Bekaert, 2002, Campbell, Forbes, Koedijk, \& Kofman, 2008, Støve, Tjøstheim, \& Hufthammer, 2014). Therefore, it is necessary to find a different approach to modeling multivariate distributions of asset returns and measuring their dependence to reach optimum portfolio construction. Modern risk management requires a measure of dependence that goes beyond simple linear correlation (Embrechts, McNeil, \& Straumann, 2002), since it is no longer sufficient to describe the dependence among the variables of interest when their joint distribution is not elliptical.

A theorem due to Sklar (1959) introduced the copula functions. According to this theorem, it is possible to decompose any $n$-dimensional joint distribution function into its $n$ marginal distributions and a copula, where the latter completely describes the dependence amongst the variables. This decomposition allows for more flexibility in the construction of multivariate distributions, which explains the major role that copulas have played in multivariate modeling lately.

Patton (2006) extended Sklar's theorem to the conditional case, defining the conditional copula, and rendered the dependence parameter conditional and time varying. Allowing for time variation in the conditional dependence among financial returns is imperative, given the evidence found in the literature that dependence among returns is not constant over time (see, for example, Patton, 2006, Christoffersen, Errunza, Jacobs, \& Langlois, 2012).

As already mentioned above, several studies investigated the asymmetry in dependence structures in international equity markets, observing that dependence tends to be high in both highly volatile markets and bear markets. These findings suggest the existence of two regimes in international equity markets: a high dependence 
regime, with low and volatile returns, and a low dependence one, with high and stable returns.

Following this conjecture, a modest literature combines the theory of conditional copulas and the Markov switching model, resulting in a sufficiently flexible framework which enables one to introduce further asymmetries in a very natural way. Jondeau and Rockinger (2006) and Silva Filho, Ziegelmann and Dueker (2012) adopt copulas with Markov switching dependence parameters. The latter allows the dependence parameter to vary according to a restricted $\operatorname{ARMA}(1,10)$ process, as in Patton $(2006)$, plus an intercept term that follows a first order Markov chain with two regimes. The former accepts that the dependence parameter assumes only two different values according to the regime. Okimoto (2008) considers more general models with Markov switching copulas, i.e., different copula functions in each regime. It is assumed therein that the copulas dependence parameters are static.

Other recent empirical investigations using Markov switching copulas include Fei, Fuertes, and Kalotychou (2017), who model the dependence between credit default swap (CDS) spreads and equity prices using bivariate copula models whose dependence parameters follow an $\operatorname{ARMA}(1,10)$ process with all coefficients changing according to a first order Markov chain, and also Stöber and Czado (2014), who find evidence of asymmetric dependence in foreign exchange markets using regime switching vine copulas.

One of the aims of this paper is to introduce an approach to modeling dependence between international financial returns over time, combining the conditional copula theory and the Markov switching model in a way that, to our knowledge, has not been explored yet. We assume different copula functions across the two regimes characterizing international equity markets, with observation driven time-varying dependence parameters. We employ these copula models and also those proposed by Patton (2006), Jondeau and Rockinger (2006) and Silva Filho et al. (2012) to model the dependence structures between the FTSE-100 returns and the CAC-40 and DAX returns, respectively. We are particularly interested in comparing these methodologies in terms of the resulting dynamics of dependence and the models' abilities to forecast possible capital losses. Because risks related to extreme events are important for portfolio construction and risk management, we compare and select the best model based on VaR forecasts for equally-weighted portfolios composed by the returns of the CAC-40 and DAX indexes in pairs with the FTSE-100 returns.

The remainder of the paper is as follows. In the next section, we briefly introduce the conditional copula theory by Patton (2006) and present the competing methodologies under consideration in this study. Following, in Section 3, we describe the estimation procedure in copula modeling in general as well as the particularities of the inferential procedures of each methodology we adopt. In Section 4, we describe the goodness-of-fit tests based on VaR forecasts. In Section 5, we first analyze the univariate return data, then, we present the estimation results and investigate the dynamics of the dependence structures, finishing with the presentation of the goodness-of-fit tests and the selection of the best models. We bring some concluding remarks in Section 6.

\section{Methodology: Conditional Copulas}

The models analyzed in this paper are based on Sklar's (1959) Theorem, extended to the conditional case in Patton (2006). According to this theorem, if $F_{1}$ is the conditional distribution of $X_{1} \mid W, F_{2}$ is the conditional distribution of $X_{2} \mid W$, and $H$ is the joint conditional distribution of $\left(X_{1}, X_{2}\right) \mid W$, where $W$ is the conditioning variable, then there exists a conditional copula $C$ such that

$$
H\left(x_{1}, x_{2} \mid w\right)=C\left(F_{1}\left(x_{1} \mid w\right), F_{2}\left(x_{2} \mid w\right) \mid w\right)
$$

where the copula contains all information about the dependence between $X_{1}$ and $X_{2}$. It means that we can decompose a (conditional) joint distribution into marginal distributions and a copula. According to Patton, it is the converse of the Sklar's theorem that is most interesting from a modeling perspective, since we can construct a joint distribution by linking together any univariate distributions with any copula.

The conditional density function corresponding to the distribution function in equation (1) can be easily recovered, provided that $F_{1}$ and $F_{2}$ are differentiable and $H$ and $C$ are twice differentiable:

$$
\begin{aligned}
h\left(x_{1}, x_{2} \mid w\right) & \equiv \frac{\partial^{2} H\left(x_{1}, x_{2} \mid w\right)}{\partial x_{1} \partial x_{2}} \\
& =\frac{\partial F_{1}\left(x_{1} \mid w\right)}{\partial x_{1}} \cdot \frac{\partial F_{2}\left(x_{2} \mid w\right)}{\partial x_{2}} \cdot \frac{\partial^{2} C\left(F_{1}\left(x_{1} \mid w\right), F_{2}\left(x_{2} \mid w\right) \mid w\right)}{\partial u_{1} \partial u_{2}} \\
& =f_{1}\left(x_{1} \mid w\right) \cdot f_{2}\left(x_{2} \mid w\right) \cdot c\left(u_{1}, u_{2} \mid w\right),
\end{aligned}
$$

where $u_{1}=F_{1}\left(x_{1} \mid w\right)$ and $u_{2}=F_{2}\left(x_{2} \mid w\right)$. This result is useful for maximum likelihood analysis, as we will see later on Section 3. 


\subsection{Observation Driven Time-Varying Copulas}

The first model we adopt is due to Patton (2006), henceforth denoted by AP copulas. Patton allows for time-variation in the conditional copula by assuming that the dependence parameter, $\theta_{c}$, evolves through time according to an equation that follows a restricted $\operatorname{ARMA}(1,10)$ process, with an autoregressive component, to capture any persistence in the dependence parameter, and a forcing variable, to capture any variation in dependence. The evolution equation of the dependence parameter may be written as

$$
\theta_{c t}=\Lambda\left(\omega+\beta \theta_{c t-1}+\alpha \psi_{t}\right)
$$

where $\Lambda$ is a logistic transformation used to keep the parameter in its interval at all times and $\psi_{t}$ is the forcing variable, defined by Patton as the mean absolute difference between the transformed marginals $u_{1 t}$ and $u_{2 t}$ over the past ten observations (Note 1). The idea is to use this measure as an indication of how far the data were from comonotonicity: if $X_{1}$ and $X_{2}$ are comonotonic, $\left|u_{1, t}-u_{2, t}\right|$ is close to zero.

We use the following copulas to analyze the dependence between the indexes considered: the elliptical copulas Normal and Student-t, and a few Archimedean copulas, namely Gumbel, Rotated-Gumbel, Clayton, Symmetrized Joe-Clayton and BB1 (Note 2). The Normal and Student-t are the copulas most frequently used in financial literature. A drawback regarding them, however, is that they describe only symmetric dependence, and it has been widely reported in literature that asymmetries are expected in financial returns, meaning that dependence in lower tail can be larger than dependence in upper tail and vice-versa. For this reason, it is also important to adopt asymmetric copulas, such as the Symmetrized Joe-Clayton and the BB1 (with lower tail dependence different from upper tail dependence), the Gumbel copula (with only upper tail dependence), and the Rotated-Gumbel and Clayton copulas (with only lower tail dependence).

\subsection{Copulas with Markov Switching}

We also adopt other three models, which combine copula theory with regime switching. In Markov switching models, introduced into econometrics by Hamilton (1989), the evolution of a time series is influenced by the different states of the world or the economy. Numerous empirical studies have found evidences that financial returns tend to exhibit different patterns of dependence according to the different states characterizing the international equity markets. More precisely, such returns tend to be more dependent during crisis periods and periods of high volatility in international equity markets than otherwise. This behavior may be described by the specification of a copula model with switching regimes.

It is possible to assume that the dependence structure of international financial returns is influenced by a hidden Markov chain with two states. For simplicity, it is assumed that this Markov chain is homogeneous and of first order such that it can be completely characterized by its transition matrix $\operatorname{Pr}\left(S_{t}=i \mid S_{t-1}=j\right)=P_{i j}$, with $i, j=0,1$, where $S_{t}$ denotes the unobserved regime at time $t$. Jondeau and Rockinger (2006) consider that the parameter $\theta_{c}$ pertaining to the dependence structure, i.e., to the copula, is driven by the following equation:

$$
\theta_{c t}=\theta_{0}\left(1-S_{t}\right)+\theta_{1} S_{t} \text {, }
$$

Where $\theta_{0}$ is the value assumed by the dependence parameter during a low-volatility/low-dependence regime and $\theta_{l}$ is the value assumed during a high-volatility/high-dependence regime and $S_{t}$ is as defined above. We will denote Jondeau and Rockinger's model by J\&R copulas.

Besides the stochastic influence on the dependence parameter, it may also be conditioned on past observations as in Silva Filho et al. (2012). The authors allow the dependence parameter to follow an $\operatorname{ARMA}(1,10)$ process, with the intercept term changing according to a Markov chain of first order and with two regimes, as described following:

$$
\theta_{c t}=\Lambda\left(\left(\omega_{0}\left(1-S_{t}\right)+\omega_{1} S_{t}\right)+\beta \theta_{c t-1}+\alpha \psi_{t}\right)
$$

where $S_{t}$ is the regime at time $t$ and $\psi_{t}$ is the forcing variable according to Patton (2006). Hereafter, this model will be denoted by SFZ\&D copulas.

We now introduce a more general approach combining time-varying copulas and regime switching, which we will denote by TZ\&SF copulas. We assume here that not only the degree of dependence changes according to the regimes characterizing international equity markets, as the type of dependence also changes, which is not the case for the two aforementioned models. It means that we assume different copula functions across the two regimes. Additionally, we allow for time-variation in the dependence parameter following Patton (2006), which differentiates our model from the one proposed by Okimoto (2008), for example, where the copula is only state dependent.

The Markov switching copula model, in this case, is specified as follows: 


$$
C_{S_{t}, t}\left(u_{1 t}, u_{2 t} \mid S_{t}, \theta_{c S_{t}, t}\right)=\sum_{k=0}^{1} \mathbf{1}_{\left\{S_{t}=k\right\}} \cdot C_{k t}\left(u_{1 t}, u_{2 t} \mid \theta_{c k t}\right),
$$

with

$$
\theta_{c k t}=\Lambda\left(\omega_{k}+\beta_{k} \theta_{c k t-1}+\alpha_{k} \psi_{t}\right)
$$

$C_{k t}(\cdot)$ is the copula function characterizing regime $k$, with time-varying dependence parameter $\theta_{c k t}, k=0,1$.

Because dependence in tails is one of the properties that discriminates between the different copulas and is relevant for both the phenomenon of asymmetric dependence and risk management, we assume copulas with different tail behaviors in each regime. Particularly, we assume that the Normal copula, which has no tail dependence, characterizes one regime, whereas an asymmetric copula prevails in the other one.

\section{Estimation Procedure: The Copula-GARCH Model}

In a time series context, we can rewrite the joint density function given in equation (2), in Section 2, as

$$
h_{t}\left(x_{1 t}, x_{2 t} \mid w, \theta\right)=f_{1 t}\left(x_{1 t} \mid w, \theta_{1}\right) \cdot f_{2 t}\left(x_{2 t} \mid w ; \theta_{2}\right) \cdot c_{t}\left(u_{1 t}, u_{2 t} \mid w ; \theta_{c}\right),
$$

where $u_{i t}=F_{i t}\left(x_{i t} \mid w ; \theta_{i}\right), i=1,2$, and $\theta=\left[\theta_{1}^{\prime}, \theta_{2}^{\prime}, \theta_{c}^{\prime}\right]$ is the vector of all parameters of both the marginals and the copula.

The expression for the log-likelihood function is as follows:

$$
\begin{gathered}
\sum_{t=1}^{T} \log h_{t}\left(x_{1 t}, x_{2 t} \mid w ; \theta\right)=\sum_{t=1}^{T} \log f_{1 t}\left(x_{1 t} \mid w ; \theta_{1}\right)+\sum_{t=1}^{T} \log f_{2 t}\left(x_{2 t} \mid w ; \theta_{2}\right)+\sum_{t=1}^{T} \log c_{t}\left(u_{1 t}, u_{2 t} \mid w ; \theta_{c}\right) \\
\ell(\theta)=\ell_{f_{1}}\left(\theta_{1}\right)+\ell_{f_{2}}\left(\theta_{2}\right)+\ell_{c}\left(\theta_{c}\right),
\end{gathered}
$$

with the maximum likelihood estimator defined as $\hat{\theta}_{M L E}=\max _{\theta \in \Theta} \ell(\theta)$.

Maximum likelihood of the joint $\log$-likelihood function $\ell(\theta)$ is possible, but this is computationally very intensive and time-consuming, especially when introducing dynamics and stochastic dependency in the model. The joint log-likelihood function (9) can be decomposed into two parts, one part involving the marginals and their parameters, and the other one involving the copula density, its parameters and also the parameters of the marginals. When the dependence is not too strong, the Inference Function for Margins (IFM) method (Joe \& Xu, 1996) can efficiently estimate the model parameters.

\subsection{The Models for the Marginal Distributions}

The estimation procedure in copula modeling begins with the identification of the marginal distributions and the estimation of their parameters via maximum likelihood. For financial return data, a univariate $\operatorname{ARMA}(\mathrm{p}, \mathrm{q})-\mathrm{GARCH}(\mathrm{m}, \mathrm{n})$ specification is usually chosen to model the marginal distributions (Note 3). It can be described by the following equations:

$$
\begin{gathered}
x_{t}=\phi_{0}+\sum_{i=1}^{p} \phi_{i} x_{t-i}+a_{t}-\sum_{j=1}^{q} \theta_{j} a_{t-j}=\mu_{t}+a_{t} \\
a_{t}=h_{t}^{1 / 2} \varepsilon_{t}, \\
h_{t}=\alpha_{0}+\sum_{i=1}^{m} \alpha_{i} a_{t-i}^{2}+\sum_{j=1}^{n} \beta_{j} h_{t-j},
\end{gathered}
$$

where $\mu_{t}$ and $h_{t}$ are the conditional mean and variance given past information, respectively. $\mathcal{E}_{t}$ is the innovation process and, in this paper, we assume that it may have a standard Normal distribution, $\varepsilon_{t} \sim \operatorname{Normal}(0,1)$, a Student-t distribution, $\varepsilon_{t} \sim$ Student $-t(v)$, or a Skewed-t distribution, $\varepsilon_{t} \sim$ Skewed $-t(v, \lambda)$. We choose the best model using the AIC and BIC and verify the goodness-of-fit using standard tests described in Section 5.2.

\subsection{Establishing a Functional Form for the Copula and Estimating the Dependence Parameter}

In the second step of the estimation procedure, the joint log-likelihood function, $\ell(\theta)$, can be reduced to the 
copula $\log$-likelihood, $\ell_{c}\left(\theta_{c}\right)$, since the $\log$-likelihoods related to the marginal distributions, $\ell_{f 1}\left(\theta_{1}\right)$ and $\ell_{f 2}\left(\theta_{2}\right)$, are fixed. The problem now is to maximize the copula log-likelihood over the copula parameter, taking the parameters of the marginals as fixed at the estimated values from the first step.

The models under analysis in this paper differ in terms of their hypotheses about the dependence structure between the financial returns, therefore, they have distinct copula log-likelihoods. For the AP copulas, $\ell_{c}$ may be written as:

$$
\ell_{c}\left(\theta_{c t}\right)=\sum_{t=1}^{T} \log c_{t}\left(u_{1 t}, u_{2 t} \mid \theta_{1}^{\text {资 }}, \theta_{2} ; \theta_{c t}\right),
$$

where $\hat{\theta}_{1}$ and $\hat{\theta}_{2}$ are the estimates of the parameters from step one, $\theta_{c t}$ is as defined in equation 3, and the $\log$-likelihood function can be maximized directly over the dependence parameter.

For the copulas with Markov switching, J\&R, SFZ\&D and TZ\&SF copulas, the log-likelihoods cannot be maximized directly over $\theta_{c}$. In order to draw inference for copula models with Markov switching, we need to overcome the challenge of having unobserved latent variables. To do so, we decompose the copula density of $u_{1 t}, u_{2 t}$ and the unobserved variable $S_{t}$ into the product of conditional and marginal densities:

$$
c_{t}\left(u_{1 t}, u_{2 t}, S_{t} \mid \omega_{t-1}\right)=c_{t}\left(u_{1 t}, u_{2 t} \mid S_{t}, \omega_{t-1}\right) \cdot \operatorname{Pr}\left(S_{t} \mid \omega_{t-1}\right),
$$

where $\omega_{t-1}$ is all information available up to time $t-1$. And, then, integrate the $S_{t}$ variable out of the joint density by summing over all possible values of $S_{t}$ :

$$
c_{t}\left(u_{1 t}, u_{2 t} \mid \omega_{t-1}\right)=\sum_{S_{t}=0}^{1} c_{t}\left(u_{1 t}, u_{2 t}, S_{t} \mid \omega_{t-1}\right)=\sum_{S_{t}=0}^{1} c_{t}\left(u_{1 t}, u_{2 t} \mid S_{t}, \omega_{t-1}\right) \cdot \operatorname{Pr}\left(S_{t} \mid \omega_{t-1}\right) .
$$

Thus, the copula log-likelihoods can be written as (Note 4)

$$
\ell_{c}=\sum_{t=1}^{T} \log \left(\sum_{S_{t}=0}^{1} c_{t}\left(u_{1 t}, u_{2 t} \mid S_{t}, w_{t-1}\right) \cdot \operatorname{Pr}\left(S_{t} \mid w_{t-1}\right)\right) .
$$

To compute the conditional probabilities $\operatorname{Pr}\left(S_{t} \mid w_{t-1}\right), S_{t}=0,1$, we apply Kim's filter, described in Kim and Nelson (1999). Being able to calculate these probabilities, we can evaluate and maximize the copula log-likelihood, obtaining the estimates of the copula parameters.

\section{Goodness-of-Fit Based on VaR Forecasts}

Because risks associated with rare (or extraordinary) events are important for the composition of portfolios, we choose to compare and select the best model based on VaR (Value at Risk) forecasts. The VaR over the time horizon $h$ with probability $\alpha, 0<\alpha<1$, can be defined as $\operatorname{VaR}_{\alpha, h}=\inf \left\{x: F_{h}(x) \geq \alpha\right\}$, where $F_{h}$ is the cumulative distribution function of the portfolio returns and $\mathrm{VaR}_{\alpha, h}$ is the ${ }^{x} 100 \alpha$-th quantile of $F_{h}$ (Note 5). In other words, the $\mathrm{VaR}$ is the maximal loss associated with the portfolio, during a certain time period (in our case, we choose a one-day period), for a given significance level $\alpha$. Although $F_{h}$ can be theoretically computed from the marginal conditional distributions, it is not easy to reach a closed analytical form for the joint distribution. For this reason, we obtain the parametric distributions of equally-weighted portfolio returns of the form $X_{P t}=0.5 X_{1 t}+0.5 X_{2 t}$ via Monte Carlo simulations and find the extreme quantiles, using the following algorithm:

For each point in time, $t=1, \ldots, T$ :

1) Simulate $K$ samples of uniforms from the fitted copula model, $\left(u_{1 t}^{(k)}, u_{2 t}^{(k)}\right), k=1, \ldots, K$, using the conditional sampling method.

2) For $k=1, \ldots, K$, convert $u_{i t}^{(k)}$ to $\varepsilon_{i t}^{(k)}, i=1,2$, using the inverse of the marginal distribution $F_{i t}$ with the estimated parameters from the first step of the IFM method, $\varepsilon_{i t}^{(k)}=F_{i t}^{-1}\left(u_{i t}^{(k)}\right)$.

3) For $k=1, \ldots, K$, convert $\varepsilon_{i t}^{(k)}$ to the $\log$-return $x_{i t}^{(k)}=\hat{\mu}_{i t}+\sqrt{h_{i t}} \cdot \varepsilon_{i t}^{(k)}$, where $\hat{\mu}_{i t}$ and $h_{i t}$ are the conditional mean and variance values, as obtained in the first step of the IFM method. 
4) For $k=1, \ldots, K$, compute the portfolio return as $x_{P t}^{(k)}=0.5 x_{1 t}^{(k)}+0.5 x_{2 t}^{(k)}$.

5) Calculate the one-day $100 \alpha$-th percentile of $x_{P t}^{(k)}, k=1, \ldots, K$, which corresponds to $\widehat{V a R}_{\alpha, 1}$. If the observed value of $X_{P t}$ for day $\mathrm{t}$ is less than $\widehat{V a R}_{\alpha, 1}$, then we say that a violation (or exceedance) occurs.

To evaluate the VaR forecasts (and their underlying models), we initially use the likelihood ratio tests proposed by Kupiec (1995) and Christoffersen (1998). Based on the previous algorithm, it is possible to construct an indicator sequence of violations $I_{t}, t=1, \ldots, T$. The unconditional coverage test, introduced by Kupiec, is a test of the null hypothesis that the indicator function $I_{t}$, which is assumed to follow an i.i.d. Bernoulli process, has a constant success probability equal to the significance level of the $V a R_{\alpha}$, where success corresponds to the portfolio losing more than the VaR. A model may pass the unconditional coverage test, i.e., the observed number of violations is close to the expected number, but we can still reject the VaR model if the violations are not independent. Christoffersen (1998) proposed a combined test for both unconditional coverage and independence, with $H_{0}$ being serial independence and a violation rate of $\alpha$.

According to Lopez (1999), the statistical tests proposed by Kupiec and Christoffersen to evaluate the accuracy of VaR models can have relatively low power against inaccurate VaR models. For this reason, he proposed an alternative methodology based not on a statistical testing framework, but instead on standard forecast evaluation techniques: the accuracy of the VaR forecasts is determined by how well they minimize a certain regulatory loss function. Simulation results indicated that this methodology is less susceptible to model misclassification and there is also the advantage of specifying the loss function according to particular interests. In light of these facts, we implement this additional procedure proposed by Lopez and we apply a test for superior predictive ability (SPA) proposed by Hansen (2005) to determine which model significantly minimizes the expected loss function.

The Basel II Accord, still in process of implementation by several countries, proposed that the capital requirements for bank's credit risk exposure were determined according to its daily VaR estimates with a $1 \%$ significance level. The capital requirement loss function (CR) is based on the larger out of the current VaR estimate and a multiple of the average estimate over the past 60 days as follows (Note 6):

$$
C R_{t}=\max \left[\frac{(3+\delta)}{60} \sum_{i=0}^{59} \operatorname{VaR}_{\alpha, t-i}, V_{a R_{\alpha, t}}\right], \delta= \begin{cases}0, & \text { if } \zeta \leq 4 \\ 0.3+0.1(\zeta-4), & \text { if } 5 \leq \zeta \leq 6 \\ 0.65, & \text { if } \zeta=7 \\ 0.65+0.1(\zeta-7), & \text { if } 8 \leq \zeta \leq 9 \\ 1, & \text { if } \zeta \geq 10\end{cases}
$$

where $\delta$ is a multiplicative factor that depends on the number of violations of the VaR in the previous 250 trading days $(\zeta)$. We adopt this regulatory loss function to evaluate the VaR forecasts.

To compare the models performances in minimizing the CR loss function, we use the SPA test statistic proposed by Hansen. A superior predictive ability test is applied whenever the interest is to test whether a particular forecasting procedure is outperformed by alternative forecasts. We are interested to know whether any of the models, $k=1, \ldots, m$, are better than a benchmark, $b c h$, in terms of minimizing the expected loss function. So we test the null hypothesis that the best model is not better than the benchmark. The relative performance of a model to the benchmark may be defined as $d_{k, t}=C R_{b c h, t}-C R_{k, t}$. Provided that $E\left(d_{k, t}\right)=\mu_{k, t}$ is well defined, we can formulate the null hypothesis of interest as

$$
H_{0}: \max _{k=1, \ldots, m} \mu_{k} \leq 0
$$

whereas the alternative hypothesis is that the best model is superior to the benchmark. A $k$ model is better than the benchmark if and only if $E\left(d_{k, t}\right)>0$. The test statistic is given by

$$
T^{S P A} \equiv \max \left[\max _{k=1, \ldots, m} \frac{T^{1 / 2} \bar{d}_{k}}{\hat{\omega}_{k}}, 0\right]
$$

where $\bar{d}_{k} \equiv T^{-1} \sum_{t=1}^{T} d_{k, t}$ and $\hat{\omega}_{k}^{2}$ is some consistent estimator of $\omega_{k}^{2} \equiv \operatorname{var}\left(T^{1 / 2} \bar{d}_{k}\right)$. The test is implemented via stationary bootstrap of Politis and Romano (1994). 


\section{Data Description, Estimations and Forecasts}

In this section, we model the dependence between the returns of the FTSE-100 index and the returns of the CAC-40 and DAX indexes, respectively, according to the methodologies presented in Section 2, analyze the dependence time dynamics and verify the goodness-of-fit on the basis of the accuracy of the VaR forecasts.

\subsection{Return Data and Descriptive Statistics}

To perform our comparative study, we use 3017 observations of daily log-returns of the stock indexes FTSE-100, CAC-40 and DAX from January 04, 1999, to April 28, 2011. We use close-to-close returns, meaning that the daily returns are those observed for trading days occurring simultaneously in all the three stock markets considered. The rationale for using only European indexes is to guarantee that the trading times overlap the most possible in order to obtain a synchronism of the returns.

The period covered by our data sample comprises two main stock market crashes worthy to be mentioned: the one of 2000 until 2002, which was a ramification of the "dot-com bubble", which burst on March 2000, and the subprime crisis, from fall 2007 to June 2009. These are periods when the markets were in a downturn trend, so we expect that the copula models capture greater dependence then. The period 2003 to 2006 is considered a period when the markets performed well.

A few descriptive statistics of the returns are provided in Table B1. We can notice that data usually shows negative asymmetry, except for CAC log-returns, suggesting that the presence of negative extreme values is more common, i.e., the left tail of the distribution is heavier. Data also presents excess kurtosis, especially the FTSE log-returns. Also, according to the Jarque-Bera test statistics, it is possible to reject the null hypothesis of normality of the returns for all indexes. All these statistics are in accordance with what is reported in the financial literature.

\subsection{Modeling the Marginal Distributions}

Here we parameterize the marginal distributions of the returns according to the ARMA-GARCH model, as described in Section 3.1. Taking into account the information criteria AIC and BIC, the diagnostic (autocorrelation) tests of the residuals and the goodness-of-fit test that the PIT of the residuals are uniforms, we choose as the best specifications for the marginals the following models: AR(3)-GARCH(1,1) with Skewed-t errors for the FTSE-100 and CAC-40 returns, and AR[4]-GARCH $(1,1)$ with Skewed-t errors for the DAX returns.

The estimates from the ARMA-GARCH fits (Note 7), the diagnostic checks and the results of the goodness-of-fit test are presented in Table B2. If the ARMA-GARCH specifications are successful at modeling the serial correlation in the conditional mean and variance, there should be no autocorrelation left in the standardized residuals and squared standardized residuals. Table B2 provides the $p$-values of the Ljung-Box test of autocorrelation in the standardized and squared standardized residuals with 15 lags, $Q(15)$ and $Q^{2}(15)$, respectively. For all series, the null hypothesis of no autocorrelation left cannot be rejected at the $5 \%$ level. Additionally, if the marginal distributions are well specified, the probability integral transforms of the standardized residuals should be $U[0,1]$. The $p$-values of the Kolmogorov-Smirnov test, reported in Table B2, suggest that the PIT of the standardized residuals have the correct distribution.

\subsection{Modeling the Dependence Structure and Analyzing its Dynamics}

Having estimated the marginal distributions, the next step is the modeling of the dependence structures between the FTSE-100 returns and the returns of CAC-40 and DAX. In this subsection, we present the copulas estimates (Note 8) and we are interested in investigating the dynamics of the dependence structures. For this purpose, we analyze the estimates of the dependence parameters and make an initial selection of the copula model that may be a good choice to represent the dependence structure of each pair of indexes based on the maximum $\log$-likelihood ( $\log \mathrm{L})$ (Note 9), observing the statistical significance of the estimated coefficients of the models. Additionally, we compare the different evolutions of the dependence measured by the Kendall's tau $(\tau)$ and the tail dependence $(\lambda)$ (Note 10), computed based on the different copulas, to provide further insight on the dependence dynamics through time.

\subsubsection{AP copulas}

We begin analyzing the estimates of the AP copulas in Table B3. For the pair FTSE-CAC, the Student-t copula is the best one out of these copulas based on the LogL and taking into account the fact that the BB1 copula does not have all estimated coefficients considered statistically significant. The estimate of $\beta_{1 T}, 4.8885$, indicates a high 
persistence in the linear correlation between FTSE and CAC. Notice that the LogLs of the Student-t and the Rotated-Gumbel copulas are very close, 2059.6 and 2058.8, respectively, so we should not just discard the Rotated-Gumbel copula. Analyzing the estimates for FTSE-DAX, we choose the Rotated-Gumbel as the best copula, according to the same criterion of highest log-likelihood amongst the models with all coefficients considered statistically significant. The Normal copula, in this case, also captures a significant persistence in the estimate of the correlation coefficient, with the estimated $\beta_{N}$ equal to 3.5251.

To observe the dependence time dynamics captured by these copulas, we focus on the evolutions of the Kendall's tau and the tail dependence. The evolution of the dependence measured by the Kendall's tau for the pair FTSE-CAC is shown in Figure C1. We have the time path of the dependence obtained from the Student-t copula in panel (a), and we also show the time paths obtained from the other copulas with all coefficients statistically significant. It is possible to notice that, from both the Student-t and the Normal copulas, the Kendall's tau evolves through time following a path that oscillates around the constant values estimated from the static versions of such copulas ( 0.6557 and 0.6496 , respectively) all along the sample. From the Rotated-Gumbel, in panel (b), the Kendall's tau remains under the estimated constant value, 0.6376, until the end of 2001, when it reaches another level and begins oscillating around this value, and, finally, it rises above this baseline from 2007 until the second semester of 2010. We expected to observe this increase in dependence after 2007 because of the subprime crisis. The subtle increase in dependence by the end of 2001 may be associated with the market crash due to the "dot-com bubble", but notice that its influence on dependence is much weaker than the effect of the subprime crisis. The evolution of the tail dependence for this same pair of indexes can be observed in Figure C2. The tail dependence path calculated based on the Student-t copula (panel (a)) is quite erratic and not very informative, remaining a little under the constant value, 0.4763, most of the time, with abrupt jumps in 2008 and 2010 associated with sudden disturbances in the estimated degrees of freedom. The lower tail dependence measured based on the Rotated-Gumbel copula, in panel (b), is greater than the one measured by the Student-t, probably because of the symmetry imposed by the elliptical functional forms, and it increases after 2007, corroborating again the expectations of greater dependence during crisis periods.

The Kendall's tau paths computed based on all statistically significant copulas for the pair FTSE-DAX are presented in Figure C3. From the Rotated-Gumbel copula, in panel (a), the Kendall's tau remains under the estimated constant value, 0.5744 , almost all the time from 1999 to the end of 2003, oscillates around this value in the period 2004 to 2006, and, from 2007 to the end of 2010, it rises above it. The same behavior is captured by the Symmetrized Joe-Clayton copula (panel (b)). In panel (c), the time path of Kendall's tau captured by the Normal copula is always oscillating around the constant value, 0.5891 . The time evolution of the tail dependence in Figure C4 is rather similar to the Kendall's tau evolution in the sense that it begins lower than its constant value, oscillates around it in the period 2004 to 2006 and finally rises above it from 2007 on. The lower tail dependence computed based on the Rotated-Gumbel is quite the same as if computed from the SJC, as can be seen from their time evolutions in panels (a) and (b), respectively. About the dependence in the upper tail computed based on the SJC, its constant value is 0.5747 , lower than the constant value for the lower tail dependence, as expected due to the typical behavior of higher dependence in lower tails than upper tails in financial markets. However, the evolution of the upper tail dependence, in panel (c), is more volatile.

From the estimation of the AP copulas, we find strong evidences of dependence between extreme values (tail dependence) for the pairs FTSE-CAC and FTSE-DAX, since the Normal copula has the lowest value for the log-likelihood amongst all copulas. These models also capture an increase in dependence beginning in 2007, when the subprime crisis was evolving, and it reaches the highest level during this period.

\subsubsection{J\&R and SFZ\&D copulas}

Table B4 reports the estimates of the J\&R copulas. We denote the probabilities $\operatorname{Pr}\left(S_{t}=1 \mid S_{t-1}=1\right)$ by $p$ and $\operatorname{Pr}\left(S_{t}=0 \mid S_{t-1}=0\right)$ by $q$. Examining the table, we select the Normal copula as the best model for the pair FTSE-CAC, noting that, though the Student-t has a higher LogL, the estimate of the degrees of freedom in regime 1 is not significant. The increase in the estimated correlation coefficient from 0.7659 in regime 0 to 0.9253 in regime 1 is strongly significant. It is also possible to conclude that the regimes are rather balanced, with very close expected durations, of $(1-p)^{-1}=98$ days for the high dependence regime and $(1-q)^{-1}=83$ days for the low dependence regime, suggesting that both of them are relevant to capture the dynamics in the dependence structure between FTSE and CAC. In fact, all the estimated copulas with significant coefficients present this same persistence in the regimes and strong significance of the dependence parameters. For the pair FTSE-DAX, we choose the Student-t copula as the best model, although with some reservations, since $p$ and $q$ are significant only at the $10 \%$ level. The estimated linear correlation increases from 0.7093 to 0.8818 , and 
the degrees of freedom do not change much, they go from 13.4763 to 14.5128 . The regimes here are very persistent, regime 1 lasts about 270 days, whereas regime 0 lasts 244 .

We analyze now the evolutions of the dependence measures $\tau$ and $\lambda$ through time to provide further insight on the dynamics of the dependence captured by these copulas with regime dependent parameters. As in Jondeau and Rockinger (2006), the paths are calculated based on the ex-ante probabilities and defined as $\tau_{t}=\tau_{0} p_{0 t}+\tau_{1}\left(1-p_{0 t}\right)$ and $\lambda_{t}=\lambda_{0} p_{0 t}+\lambda_{1}\left(1-p_{0 t}\right)$, where $p_{0 t}=\operatorname{Pr}\left(S_{t}=0 \mid w_{t-1}\right)$. In Figure C5, we plot $\tau_{t}$ for FTSE-CAC and it reveals that the dependence between these indexes is characterized essentially by three subperiods. The first one, that goes from 1999 to the first semester of 2001, is mainly associated with the low dependence regime, with two short-lasting increases in dependence in 1999 and 2001. In the second period, beginning by the end of 2001 and going until 2006, the two regimes are intercalated. From the second semester of 2001 to the end of 2002, the higher dependence can be explained by the market crash due to the "dot-com bubble", besides of the terrorist attack to the Twin Towers on September, 11, 2001. Unexpectedly, these copulas capture increases in dependence during the period 2003 to 2006. This is a surprise because it is a period when the international financial markets performed well. Finally, the third period, from 2007 on, is associated with the high dependence regime, when the two markets are strongly dependent. This period of high dependence coincides with the subprime crisis until the first semester of 2009 and goes beyond it until the end of 2010, and then the dependence decreases in the beginning of 2011. During this period, there is a short-lasting decrease in dependence in the first semester of 2008, probably associated with an increase in the economic activity of the US identified by the Business Cycle Dating Committee of the National Bureau of Economic Research. Figure C6 displays the evolution of the tail dependence, whose pattern is similar to the one described above for the Kendall's tau. From the BB1 copula, in panel (a), $\lambda_{L}$ is lower than $\lambda_{U}$ during regime 0, and higher than this during regime 1. This behavior is reverted when the tail dependence is computed based on the Symmetrized Joe-Clayton copula (panel (c)).

In Figure C7, we have the Kendall's tau evolution for the pair FTSE-DAX. There we can identify two long subperiods characterizing the dependence between these two indexes. From 1999 to January, 2004, the dependence is mainly low, with a significant increase by the end of 2002 and beginning of 2003. The second subperiod, from 2004 on, is essentially associated with the high dependence regime. During this period, an important decrease in dependence occurs in the second semester of 2005 and it rises again from 2006 to the end of 2010. Just as it happened for FTSE-CAC, the dependence between FTSE and DAX increased during the crises of 2002 and 2007-2009. Tail dependence through time is displayed in Figure C8. The lower and upper tail dependence parameters calculated based on the Student-t copula (panel (a)), assume the value 0.1389 in regime 0 and 0.3367 in regime 1 , suggesting that both the dependence between extreme negative values and the dependence between extreme positive values increase during turbulent periods such as 2002 and 2007 to 2009 . The asymmetric copulas indicate much higher extreme dependence, ranging from 0.4 to 0.55 in regime 0 and 0.6 to 0.75 in regime 1 . Based on the BB1 copula (panel (b)), $\lambda_{L}$ is lower than $\lambda_{U}$ in regime 0 , and higher than this in regime 1. Based on the Symmetrized Joe-Clayton (panel (d)), $\lambda_{L}$ is higher than $\lambda_{U}$ all along their paths.

The estimated SFZ\&D copulas are provided in Table B5. According to the log-likelihood criterion, we choose the Student-t copula as the best model for both the pairs FTSE-CAC and FTSE-DAX. Note that, differently from the other copulas with two parameters, we assume, in the case of the Student-t, that the degrees of freedom are only regime dependent, they do not follow an $\operatorname{ARMA}(1,10)$ process. The reason is that the estimation of the Student-t copula is very time-consuming and, with this assumption, we intend to make this task a little easier. The estimates of the correlation coefficient for both pairs are very persistent, with the estimated $\beta_{1 T}$ equal to 3.3971 and 2.3508 for FTSE-CAC and FTSE-DAX, respectively. The intercept term of the estimated correlation coefficient and the estimated degrees of freedom increase significantly from regime 0 to regime 1 for both pairs of indexes. The regimes are balanced and very persistent: regime 1 lasts 128 days and regime 0 lasts 122, for FTSE-CAC, whereas the expected durations are 555 and 500 days for regimes 1 and 0 , respectively, for FTSE-DAX.

To scrutinize the dynamics of the dependence structure between FTSE and CAC captured by these models, we look at Figure C9. Interestingly, we notice that the Kendall's tau path estimated based on the Student-t copula (panel (a)) displays a rather similar pattern to the Kendall's tau path computed based on the Normal J\&R copula (Figure C5, panel (a)). This corroborates the close values of the LogLs obtained for the two models (2147.4 and 2128.8 , respectively). The Student-t SFZ\&D copula does not indicate some short-lasting increases in dependence in 1999, beginning of 2001 and second semester of 2003, as the Normal J\&R copula does, but the models do 
coincide in determining the regimes for the rest of the sample. Still examining Figure $\mathrm{C} 9, \tau_{t}$ in panel (c), related to the Symmetrized Joe-Clayton copula, behaves similarly to the $\tau_{t}$ in panel (a), related to the Student-t, but the evolution of the dependence measured based on the Rotated-Gumbel, in panel (b), suggests more unbalanced regimes. The patterns of evolution of the tail dependence parameters from the copulas Student-t, Rotated-Gumbel and SJC in Figure C10 mimic their counterparts in Figure C9. Once again, the tail dependence captured by the asymmetric copulas is higher than that captured by the Student-t, and $\lambda_{L}$ is higher than $\lambda_{U}$ in the regime of high dependence, based on the SJC copula.

For FTSE and DAX, the evolution of the Kendall's tau computed based on the Student-t copula, in Figure C11, panel (a), suggests that the dependence between these indexes is characterized by two subperiods, one of low dependence, from 1999 to January, 2004, and another of high dependence, from 2004 on, just as the Student-t J\&R copula, in Figure C7, panel (a), indicates. Although, this time, the regimes are even more persistent: the current copula does not indicate a significant increase in dependence by the end of 2002 and beginning of 2003, besides of other short-lasting increases during the period 1999 to 2004, and it does not capture a decrease in dependence in the first semester of 2005, as the other model does. This behavior of the dependence confirms the close values of the LogLs of the two models, 1683.8 for the Student-t SFZ\&D copula, and 1671.1 for the Student-t J\&R copula. Differently from the results obtained from the elliptical copulas, the asymmetric copulas BB1 and Rotated-Gumbel in Figure C11 suggest that regime 0 is more persistent than regime 1 . The tail dependence computed based on the current Student-t copula, Figure C12, panel (a), does not differ substantially from that based on the Student-t J\&R copula: on average, it measures 0.1490 in regime 0 and 0.3285 in regime 1. Curiously, the tail dependence parameters based on the BB1 copula, panel (b), are more volatile in the low dependence regime than in the high dependence one.

The estimation results of the J\&R and SFZ\&D copulas indicate, for both pairs of indexes, the prevalence of a long period of high dependence beginning in 2007, for FTSE-CAC, and 2006, for FTSE-DAX, until the end of 2010. This period coincides with or slightly precedes the beginning of the subprime crisis and goes beyond it. For FTSE and CAC, these copulas also capture significant increases in dependence by the end of 2001 and 2002, whereas for FTSE and DAX, not all SFZ\&D copulas capture increases in dependence during the crash of 2000 to 2002. Surprisingly, these copulas also suggest increases in dependence during the period 2003 to 2006, when the conditions in the international financial markets were favorable. In addition, we can notice that, for these models, the elliptical copulas have a better fit to the data. The tail dependence, when it exists, is probably symmetrical, given the better fit of the Student-t copula.

\subsubsection{TZ\&SF Copulas}

Finally, Table B6 presents the estimates of the TZ\&SF copulas. For the pair FTSE-CAC, the only model with all coefficients statistically significant is the combination Normal and Clayton. In the estimation of the combinations with the SJC, Rotated-Gumbel and Gumbel copulas, all coefficients related to the asymmetric copulas are statistically not significant, except for the estimate of $w_{R G}$. For the model Normal and BB1, the estimates of $\beta_{2 b b 1}$ and $\alpha_{1 b b 1}$ are not significant, indicating lack of autocorrelation in the parameter $\kappa$ and no influence of the combined movement of the marginal probability transforms in the path of $\gamma$. For the pair FTSE-DAX, none of the combinations has all coefficients statistically significant: for the Normal-Clayton and the Normal-SJC, coefficients related to the Normal copula are not significant, whereas for Normal and Gumbel and Normal and Rotated-Gumbel, coefficients related to the asymmetric copula have no statistical significance. The Normal-BB1 combination has almost all coefficients statistically not significant.

Figure C13 displays, in panel (a), the evolution of the Kendall's tau for the pair FTSE-CAC, computed based on the Normal-Clayton Markov switching copula, and the smoothed probabilities of the high and low dependence regimes, in panels (b) and (c), respectively. As before, we can identify here three subperiods characterizing the dependence between FTSE and CAC. Until the first semester of 2001, we have mainly low dependence between the indexes. From the end of 2001 to the second semester of 2006, the regimes are intercalated, however, now, the low dependence periods are much shorter than before. The low dependence regime has expected duration of 20 days, in contrast with the expected duration of 98 days of the high dependence regime. From 2007 onwards, high dependence prevails. Notice that the two regimes are characterized by different tail behaviors, with no tail dependence at all in the high dependence regime, and lower tail dependence in the low dependence one, which is, actually, opposite to what we expected to find.

\subsection{Forecasting VaR for Goodness-of-Fit Check and Model Selection}

We proceed now to the evaluation of the VaR forecasts based on the estimated copula models. For the VaR 
computation, we simulate 1000 replications of the portfolio returns for each point in time from the estimated copulas with all coefficients statistically significant. We first evaluate the forecasts by applying the likelihood ratio tests proposed by Kupiec and Christoffersen. Then, we compute the (capital requirement) losses based on the $1 \%$ daily VaR forecasts from those models that passed the first tests, and apply the SPA test to determine which model has a superior predictive ability.

We first analyze the forecasts from the AP copulas. Table B7 presents the results of the Kupiec and Christoffersen tests for the daily VaR forecasts. One can see from this table that, for both pairs of indexes, the AP copulas have good performances in terms of forecasting, since their $1 \%$ and $5 \%$ VaR forecasts pass both likelihood ratio tests of unconditional coverage and combination of coverage and independence. Notice that the $\mathrm{VaR}$ forecasts at the $1 \%$ significance level are aggressive, meaning that the forecasted quantile is more extreme than the theoretical VaR, and, at the 5\% level, they are more conservative, except for the Rotated-Gumbel forecasts for the pair FTSE-CAC. The average losses computed based on the 1\% daily VaR forecasts and the results of the SPA test (Note 11) are presented in Table B8. We implement the SPA test considering each copula at a time as the benchmark and the remaining ones as the competing alternatives. For the AP copulas, for both pairs of indexes, it is not possible to reject the null hypothesis that the best competing copula cannot outperform the Normal copula.

Table B7 also reports the results of the likelihood ratio tests for the VaR forecasts from the J\&R copulas. In this case, with the exception of the BB1 copula, with too aggressive $1 \%$ daily VaR forecasts, the other copulas pass the Kupiec and Christoffersen tests of the VaR forecasts accuracy. Also for these models, the 1\% VaR forecasts are more aggressive than the 5\% VaR forecasts, considered quite conservative, except for the Rotated-Gumbel forecasts. In terms of minimizing the regulatory loss function, the results in Table B8 for the J\&R copulas suggest that no alternative copula can outperform the Normal copula for both the FTSE-CAC and FTSE-DAX pairs.

Finally, from the results of the likelihood ratio tests for the forecasts based on the SFZ\&D copulas, presented in Table B7, it is possible to state that the BB1 copula is the only one that cannot appropriately approximate the joint distribution of the FTSE and DAX returns, at least, not in its tails. The results of the SPA test for the SFZ\&D copulas, provided in Table B8, indicate that the Student-t performs best in terms of forecasting for the pair FTSE-CAC, whereas the Normal copula is the best choice for FTSE and DAX.

Having selected, for both pairs of indexes, the models with the best performances in forecasting out of each methodology, we now apply the SPA test to them, to finally find the one that has the best fit to the data in the lower tail of the joint distribution of the returns. In the case of FTSE-CAC, we also consider the Normal-Clayton Markov switching copula, since it is the only model remaining from the TZ\&SF copulas, and it passes the Kupiec and Christoffersen tests, with $p$-values 0.6048 and 0.5848 , respectively, for the VaR forecasts at the $1 \%$ level, and 0.3483 and 0.5506 , for the 5\% VaR forecasts. Table B9 displays the results of this test. It is not possible to reject the null hypothesis that the best alternative model cannot outperform the Normal J\&R copula in the forecasting of the first percentile of the FTSE-CAC portfolio returns. At the same time, one should notice that, at a lower significance level, of $1 \%$, it is not possible to reject the null that the best alternative model is not better than the Normal AP copula, when this one is assumed as the benchmark. In this case, we could assert that both models provide statistically similar predictions of the extreme (negative) quantiles of the FTSE-CAC portfolio. However, the last result is not robust to alternative choices of the block length for the bootstrap. So, we conclude that the Normal J\&R copula provides the best VaR forecasts for the portfolio composed by FTSE and CAC, noticing that, amongst the best forecasts, these are the most aggressive ones. For the portfolio formed by FTSE and DAX, the SPA test indicates that no alternative model has a better performance in terms of forecasting the extreme quantiles than the Normal AP copula. Although, at the $1 \%$ significance level, we could accept that the Normal SFZ\&D copula provides similar forecasts. Nevertheless, this result changes when we assume different block lengths. Hence, according to our results, the Normal AP copula performs best in forecasting the VaR for the portfolio composed by FTSE and DAX, with less aggressive predictions.

\section{Concluding Remarks}

In this paper, we introduce a new approach to modeling dependence between financial return data over time, combining time-varying copulas and the Markov switching model, and we employ this methodology and also those proposed by Patton (2006), Jondeau and Rockinger (2006) and Silva Filho et al. (2012) to model the dependence structures between the FTSE-100 returns and the returns of the indexes CAC-40 and DAX, respectively, over the period 1999 to April 2011. We also use the copula estimates to carry out tail inferences and find out the copula model with the best performance in forecasting the extreme quantiles for each portfolio 
composed by the pairs of indexes aforementioned.

Based on the log-likelihood criterion, we find strong evidences of tail dependence for the pairs FTSE-CAC and FTSE-DAX from the estimation of the AP copulas, since the Normal copula has the lowest value for the log-likelihood amongst all copulas. For FTSE and DAX, the tail dependence is asymmetric, with stronger lower tail. On the other hand, analyzing the estimation results of the J\&R and SFZ\&D copulas, the elliptical copulas have a better fit to the data according to the same criterion, suggesting that, the tail dependence, when it exists, is probably symmetrical, given the better fit of the Student-t copula. We barely can find empirical evidences of time-varying copulas with distinct tail behaviors characterizing the dependence between the returns according to the international equity markets regimes. The combination of a high dependence regime characterized by the time-varying Normal copula and a low dependence regime associated with the time-varying Clayton copula for FTSE and CAC, though statistically significant, does not show a good fit to the data.

The AP copulas as well as the J\&R and SFZ\&D copulas identify a long period of high dependence from 2007 to the end of 2010, for both the pairs FTSE-CAC and FTSE-DAX. This period coincides with or slightly precedes the beginning of the subprime crisis and goes beyond it. The copula models with regime switching also capture significant increases in dependence during the period 2001 to 2002, especially between FTSE and CAC, which can be explained by the market crash due to the "dot-com bubble", besides of the so called September 11 effect. Unexpectedly, these models also indicate increases in dependence during the period 2003 to 2006, when the financial markets did well.

Because in risk management the main concern has to do with tail risks, we compare and select the copula models based on VaR forecasts. To evaluate the accuracy of these predictions, we apply the traditional Kupiec and Christoffersen tests, besides of an alternative methodology based on standard forecast evaluation techniques, i.e, the accuracy of the VaR forecasts is determined by how well they minimize a certain regulatory function. We also apply the SPA test by Hansen (2005) to determine which copula model significantly minimizes the expected loss function. Based on the results of the SPA test, we cannot reject the null hypothesis that the best alternative model cannot outperform the Normal J\&R copula in the forecasting of the extreme (negative) quantiles of the FTSE-CAC portfolio returns. This result suggests that the high persistence in the linear correlation coefficient between FTSE and CAC captured by the Student-t and Normal AP copulas is inappropriate, and the long-memory feature of this dependence parameter is, in fact, a consequence of a model with large but infrequent breaks, as the Markov switching model. Also according to the results of the SPA test, no alternative copula model can outperform the Normal AP copula in the forecasting of the VaR losses for the portfolio composed by FTSE and DAX returns. Surprisingly, the Normal copula has the best fit to the tails of the joint distributions of the returns, which is in contrast to the evidences we expected to find for financial return data and also to the evidences found in the literature of static copulas, where usually copulas that feature tail dependence and asymmetry show better fits. It seems that part of the asymmetry may be generated by time-varying parameters. Also, the lack of tail dependence may be partially compensated by the possibility of large overall dependence, which would explain why the Gaussian copula fits the data so well.

\section{Acknowledgments}

Flavio A. Ziegelmann is partially supported by a CNPq grant (process 306347/2015-6). Osvaldo Candido is also partially supported by CNPq grants (processes 453993/2014-1 and 307491/2016-1).

\section{References}

Ang, A., \& Bekaert, G. (2002). International asset allocation with regime shifts. The Review of Financial Studies, 15, 1137-1187. https://doi.org/10.1093/rfs/15.4.1137

Campbell, R., Forbes, C., Koedijk, K., \& Kofman, P. (2008). Increasing correlations or just fat tails? Journal of Empirical Finance, 15, 287-309. https://doi.org/10.1016/j.jempfin.2007.01.001

Christoffersen, P. (1998). Evaluating interval forecasts. International Economic Review, 39, 841-862. https://doi.org/10.2307/2527341

Christoffersen, P., Errunza, V., Jacobs, K., \& Langlois, H. (2012). Is the potential for international diversification disappearing? A dynamic copula approach. The Review of Financial Studies, 25, 3711-3751. https://doi.org/10.1093/rfs/hhs104

Embrechts, P., McNeil, A., \& Straumann, D. (2002). Correlation and dependence properties in risk management: Properties and pitfalls. In Dempster, M. (Ed.), Risk Management: Value at Risk and Beyond (pp. 176-223). Cambridge University Press. https://doi.org/10.1017/CBO9780511615337.008

Fei, F., Fuertes, A. M., \& Kalotychou, E. (2017). Dependence in credit default swap and equity markets: 
Dynamic copula with Markov-switching. International Journal of Forecasting, 33, 662-678. https://doi.org/10.1016/j.ijforecast.2017.01.006

Hamilton, J. D. (1989). A new approach to the economic analysis of nonstationary time series and the business cycle. Econometrica, 57, 357-384. https://doi.org/10.2307/1912559

Hansen, P. R. (2005). A test for superior predictive ability. Journal of Business and Economic Statistics, 23, 365-380. http://dx.doi.org/10.1198/073500105000000063

Joe, H., \& Xu, J. (1996). The estimation method of inference functions for margins for multivariate models. Technical report 166, Department of Statistics, University of British Columbia.

Jondeau, E., \& Rockinger, M. (2006). The Copula-GARCH model of conditional dependencies: An international stock market application. Journal of International Money and Finance, 25, 827-853. https://doi.org/10.1016/j.jimonfin.2006.04.007

Kim, C. J., \& Nelson, C. R. (1999). State-space models with regime switching: Classical and Gibbs-sampling approaches with applications. MIT Press.

Kupiec, P. (1995). Techniques for verifying the accuracy of risk measurement models. Journal of Derivatives, 3(2), 73-84. https://doi.org/10.3905/jod.1995.407942

Longin, F., \& Solnik, B. (2001). Extreme correlation of international equity markets. Journal of Finance, 56, 649-676. https://doi.org/10.1111/0022-1082.00340.

Lopez, J. A. (1999). Regulatory evaluation of Value-at-Risk models. Journal of Risk, 1, 37-64. https://doi.org/10.21314/JOR.1999.005

Okimoto, T. (2008). New evidence of asymmetric dependence structures in international equity markets. Journal of Financial and Quantitative Analysis, 43, 787-816. https://doi.org/10.1017/S0022109000004294

Patton, A. J. (2006). Modelling Asymmetric Exchange Rate Dependence. International Economic Review, 47, 527-556. https://doi.org/10.1111/j.1468-2354.2006.00387.x

Politis, D. N., \& Romano, J. P. (1994). The stationary bootstrap. Journal of the American Statistical Association, 89, 1303-1313. https://doi.org/10.1080/01621459.1994.10476870

Politis, D. N., \& White, H. (2004). Automatic block-length selection for the dependent bootstrap. Econometric Reviews, 23(1), 53-70. https://doi.org/10.1081/ETC-120028836

Sancetta, A., \& Satchell, S. E. (2001). Bernstein approximations to the copula function and portfolio optimization. Working Papers in Economics 0105, Faculty of Economics, University of Cambridge, Cambridge.

Silva Filho, O. C., Ziegelmann, F. A., \& Dueker, M. J. (2012). Modeling dependence dynamics through copulas with regime switching. Insurance: Mathematics and Economics, 50(3), 346-356. https://doi.org/10.1016/j.insmatheco.2012.01.001

Sklar, A. (1959). Fonctions de répartition a n dimensions et leurs marges. Publications de l'Institut de Statistique de L'Université de Paris, 8, 229-231.

Stöber, J., \& Czado, C. (2014). Regime switches in the dependence structure of multidimensional financial data. Computational Statistics and Data Analysis, 76, 672-686. https://doi.org/10.1016/j.csda.2013.04.002.

Støve, B., Tjøstheim, D., \& Hufthammer, K. O. (2014). Using local gaussian correlation in a nonlinear re-examination of financial contagion. Journal of Empirical Finance, 25, 62-82. https://doi.org/10.1016/j.jempfin.2013.11.006

\section{Notes}

Note 1. This is the definition for non-elliptical copulas. For the elliptical ones, the forcing variable is defined as the mean of the product $\Phi^{-1}\left(u_{1, t-j}\right) \cdot \Phi^{-1}\left(u_{2, t-j}\right)$, for the Gaussian copula, and the mean of $T_{v}^{-1}\left(u_{1, t-j}\right) \cdot T_{v}^{-1}\left(u_{2, t-j}\right)$, for the Student-t, over the previous 10 lags, where $\Phi^{-1}$ is the inverse of the standard Normal c.d.f. and $T_{v}^{-1}$ is the inverse of the Student-t c.d.f. with $v$ degrees of freedom. If data is positively dependent, the inverse of the marginal transforms of both variables will have the same sign, thus, $\alpha$ is expected to be positive. 
Note 2. Their functional forms as well as the evolution equations of their dependence parameters following Patton (2006) are described in Appendix A.

Note 3. Extensions of the GARCH model, such as EGARCH, TARCH, among others, are also fitted to data in order to find out the best model for the marginals.

Note 4. Just to keep the notation in accordance with the one used up to this point, note that, in the case that the functional form of the copula density changes according to the regime, the copula density function should be denoted by $c_{S_{t}, t}(\cdot)$.

Note 5. Since the VaR is calculated here from the distribution of the portfolio daily log-returns, it is expressed in percentage.

Note 6. Note that, since the VaR is a negative value, to compute the loss function, it will be calculated here as minus the ( $100 \alpha$-th percentile) of the c.d.f. of the returns.

Note 7. All marginals were estimated using the Oxford MFE Toolbox by Kevin Shepard.

Note 8. The copula models were estimated using the Copula Toolbox provided by Andrew Patton and some functions written by the authors for the Matlab $7^{\circledR}$ software. The standard errors were computed numerically using the functions "Hessian2sided" and "MyFuncScores" from Dynamic Copula Toolbox 3.0 provided by Manthos Vogiatzoglou.

Note 9. Since our data sample is quite long, the information criteria AIC and BIC choose the same model as the LogL. For lack of space, these information criteria are not presented here, but they are available from the authors upon request.

Note 10. The Kendall's tau measures a particular dependence known as concordance. The tail dependence measures the dependence in extreme values: the lower tail dependence, $\lambda_{L}$, (upper tail dependence, $\lambda_{U}$ ) is the probability that one variable takes an extremely large negative (positive) value, given that the other variable took an extremely large negative (positive) value.

Note 11. For the stationary block bootstrap, we use 10000 re-samples and select the optimum block length in accordance with Politis and White (2004). We use the Matlab code "opt_block_length_REV_dec07" compiled by Andrew Patton to implement the automatic block length selection.

\section{Appendix A}

\section{Copula Functions}

Normal copula: the Normal copula, extracted from the bivariate Normal distribution, is defined as follows:

$$
C_{N}\left(u_{1}, u_{2} \mid \rho\right)=\int_{-\infty}^{\Phi^{-1}\left(u_{1}\right)} \int_{-\infty}^{\Phi^{-1}\left(u_{2}\right)} \frac{1}{2 \pi \sqrt{\left(1-\rho^{2}\right)}} \exp \left\{\frac{-\left(r^{2}-2 \rho r s+s^{2}\right)}{2\left(1-\rho^{2}\right)}\right\} d r d s, \rho \in(-1,1),
$$

where the dependence parameter, $\rho$, is the linear correlation coefficient. Its dynamic equation may be written as

$$
\rho_{t}=\Lambda\left(\omega_{N}+\beta_{N} \rho_{t-1}+\alpha_{N} \cdot \frac{1}{10} \sum_{j=1}^{10} \Phi^{-1}\left(u_{1, t-j}\right) \cdot \Phi^{-1}\left(u_{2, t-j}\right)\right) .
$$

$\Lambda(\cdot)$ and $\tilde{\Lambda}(\cdot)$, which appear hereafter, are logistic transformations to keep the parameters in their intervals.

The Normal copula is symmetric and has no tail dependence, that is, $\lambda_{L}=\lambda_{U}=0$. The Kendall's tau may be computed based on the correlation coefficient as $\tau=(2 / \pi) \arcsin \rho$.

Student-t copula: it is associated with the bivariate Student-t distribution and has the following functional form:

$$
C_{T}\left(u_{1}, u_{2} \mid \rho, v\right)=\int_{-\infty}^{t_{v}^{-1}\left(u_{1}\right)} \int_{-\infty}^{t_{\nu}^{-1}\left(u_{2}\right)} \frac{1}{2 \pi \sqrt{1-\rho^{2}}}\left(1+\frac{r^{2}-2 \rho r s+s^{2}}{v\left(1-\rho^{2}\right)}\right)^{-\frac{v+2}{2}} d r d s,
$$

where the parameters $\rho$ and $v$ are the linear correlation coefficient and the degrees of freedom, respectively. In addition, their evolution equations are given by

$$
\rho_{t}=\Lambda\left(\omega_{1 T}+\beta_{1 T} \rho_{t-1}+\alpha_{1 T} \cdot \frac{1}{10} \sum_{j=1}^{10} T_{\nu}^{-1}\left(u_{1, t-j}\right) \cdot T_{\nu}^{-1}\left(u_{2, t-j}\right)\right)
$$


and

$$
v_{t}=\tilde{\Lambda}\left(\omega_{2 T}+\beta_{2 T} v_{t-1}+\alpha_{2 T} \cdot \frac{1}{10} \sum_{j=1}^{10} T_{v}^{-1}\left(u_{1, t-j}\right) \cdot T_{v}^{-1}\left(u_{2, t-j}\right)\right)
$$

The Student-t copula has symmetrical tail dependence, with $\lambda_{L}=\lambda_{U}=2 T_{v+1}\left(-\sqrt{\frac{(v+1)(1-\rho)}{1+\rho}}\right)$, where $T_{v+1}$ is the Student-t c.d.f with $(v+1)$ degrees of freedom. The Kendall's tau is given by $\tau=(2 / \pi) \arcsin \rho$.

Gumbel copula: it has the form of

$$
C_{G}\left(u_{1}, u_{2} \mid \theta\right)=\exp \left(-\left(\left(-\log u_{1}\right)^{\theta}+\left(-\log u_{2}\right)^{\theta}\right)^{1 / \theta}\right), \quad \theta \in[1, \infty) .
$$

The dynamics is given by the following equation governing the dependence parameter evolution:

$$
\theta_{t}=\Lambda\left(\omega_{G}+\beta_{G} \theta_{t-1}+\alpha_{G} \cdot \frac{1}{10} \sum_{j=1}^{10}\left|u_{1, t-j}-u_{2, t-j}\right|\right)
$$

The Gumbel copula exhibits only upper tail dependence, with $\lambda_{U}=2-2^{1 / \theta}$. It can be shown that the Kendall's tau is given by $\tau=1-\theta^{-1}$.

Rotated-Gumbel copula: or Survival Gumbel copula, which is the complement ("Probability of survival") of the Gumbel copula. It has the following form:

where $C_{G}$ corresponds to the Gumbel copula.

$$
C_{R G}\left(u_{1}, u_{2} \mid \theta\right)=u_{1}+u_{2}-1+C_{G}\left(1-u_{1}, 1-u_{2} \mid \theta\right),
$$

The dependence parameter, $\theta$, follows the process

$$
\theta_{t}=\Lambda\left(\omega_{R G}+\beta_{R G} \theta_{t-1}+\alpha_{R G} \cdot \frac{1}{10} \sum_{j=1}^{10}\left|u_{1, t-j}-u_{2, t-j}\right|\right)
$$

The Rotated-Gumbel copula has only lower tail dependence, given by $\lambda_{L}=2-2^{1 / \theta}$, and the Kendall's tau may be computed as $\tau=1-\theta^{-1}$.

Clayton copula: or Kimeldorf-Sampson copula, has the following distribution function:

$$
C_{C}\left(u_{1}, u_{2} \mid \delta\right)=\left(u_{1}^{-\delta}+u_{2}^{-\delta}-1\right)^{-1 / \delta}, \quad \delta \in(0, \infty) .
$$

The evolution equation of the dependence parameter is

$$
\delta_{t}=\Lambda\left(\omega_{C}+\beta_{C} \delta_{t-1}+\alpha_{C} \cdot \frac{1}{10} \sum_{j=1}^{10}\left|u_{1, t-j}-u_{2, t-j}\right|\right) .
$$

This copula exhibits only lower tail dependence, $\lambda_{L}=2^{-1 / \delta}$. The Kendall's tau has the form $\tau=\delta /(\delta+2)$.

Symmetrized Joe-Clayton copula: this copula was defined by Patton (2006) and takes the form of

$$
C_{S J C}\left(u_{1}, u_{2} \mid \lambda_{U}, \lambda_{L}\right)=0.5 \cdot\left(C_{J C}\left(u_{1}, u_{2} \mid \lambda_{U}, \lambda_{L}\right)+C_{J C}\left(1-u_{1}, 1-u_{2} \mid \lambda_{U}, \lambda_{L}\right)+u_{1}+u_{2}-1\right)
$$

where $C_{J C}$ is the Joe-Clayton copula, also called BB7 copula (Joe, 1997), given by

$$
C_{J C}\left(u_{1}, u_{2} \mid \lambda_{U}, \lambda_{L}\right)=1-\left(1-\left\{\left[1-\left(1-u_{1}\right)^{\kappa}\right]^{-\gamma}+\left[1-\left(1-u_{2}\right)^{\kappa}\right]^{-\gamma}-1\right\}^{-1 / \gamma}\right)^{-1 / \kappa},
$$

with $\kappa=1 / \log _{2}\left(2-\lambda_{U}\right), \gamma=-1 / \log _{2}\left(\lambda_{L}\right)$ and $\lambda_{U}, \lambda_{L} \in(0,1)$.

The SJC copula has upper and lower tail dependence and its dependence parameters are the upper and lower tail dependence parameters, $\lambda_{U}$ and $\lambda_{L}$, respectively. Furthermore, $\lambda_{U}$ and $\lambda_{L}$ range freely and are not dependent on each other. Since this copula nests symmetry as a special case, it is a more interesting specification than the BB7 copula. The evolution equations for the parameters $\lambda_{U}$ and $\lambda_{L}$ are 


$$
\lambda_{U t}=\Lambda\left(\omega_{U}+\beta_{U} \lambda_{U t-1}+\alpha_{U} \cdot \frac{1}{10} \sum_{j=1}^{10}\left|u_{1, t-j}-u_{2, t-j}\right|\right)
$$

and

$$
\lambda_{L t}=\Lambda\left(\omega_{L}+\beta_{L} \lambda_{L t-1}+\alpha_{L} \cdot \frac{1}{10} \sum_{j=1}^{10}\left|u_{1, t-j}-u_{2, t-j}\right|\right) .
$$

The Kendall's tau, in this case, has no closed form, so we compute it numerically.

BB1 copula (Joe, 1997): it has the following functional form:

$$
C_{b b 1}\left(u_{1}, u_{2}, \kappa, \gamma\right)=\left\{1+\left[\left(u_{1}^{-\kappa}-1\right)^{\gamma}+\left(u_{2}^{-\kappa}-1\right)^{\gamma}\right]^{1 / \gamma}\right\}^{-1 / \kappa}, \quad \kappa \in(0, \infty), \gamma \in[1, \infty) .
$$

The dynamic equations of the dependence parameters are

$$
\begin{aligned}
& \kappa_{t}=\Lambda\left(\omega_{1 b b 1}+\beta_{1 b b 1} \kappa_{t-1}+\alpha_{1 b b 1} \cdot \frac{1}{10} \sum_{j=1}^{10}\left|u_{1, t-j}-u_{2, t-j}\right|\right) \\
& \gamma_{t}=\tilde{\Lambda}\left(\omega_{2 b b 1}+\beta_{2 b b 1} \gamma_{t-1}+\alpha_{2 b b 1} \cdot \frac{1}{10} \sum_{j=1}^{10}\left|u_{1, t-j}-u_{2, t-j}\right|\right) .
\end{aligned}
$$

The BB1 copula has upper and lower tail dependence given by $\lambda_{U}=2-2^{1 / \gamma}$ and $\lambda_{L}=2^{-1 / \gamma \kappa}$, respectively. The Kendall's tau may be calculated based on $\kappa$ and $\gamma$ as $\tau=1-(2 /(\gamma(\kappa+2)))$.

\section{Appendix B}

\begin{tabular}{|c|c|c|c|}
\hline \multirow[b]{2}{*}{ Parameter } & \multicolumn{3}{|c|}{ Conditional Mean and Variance Equations } \\
\hline & FTSE & CAC -40 & DAX \\
\hline \multirow[t]{2}{*}{$\phi_{1}$} & -0.0577 & -0.0369 & $\ldots$ \\
\hline & $(0.0002)$ & $(0.0013)$ & $\ldots$ \\
\hline \multirow[t]{2}{*}{$\phi_{2}$} & -0.0637 & -0.0440 & $\ldots$ \\
\hline & $(0.0005)$ & $(0.0002)$ & $\ldots$ \\
\hline \multirow[t]{2}{*}{$\phi_{3}$} & -0.0731 & -0.0626 & $\ldots$ \\
\hline & $(0.0006)$ & $(0.0021)$ & \\
\hline$\phi_{4}$ & $\ldots$ & $\ldots$ & 0.0444 \\
\hline \multirow[t]{2}{*}{$\alpha_{0}$} & $1.1588 \mathrm{E}-06$ & $1.5820 \mathrm{E}-06$ & $1.7875 \mathrm{E}-06$ \\
\hline & $(0.0000)$ & $(0.0000)$ & $(0.0000)$ \\
\hline \multirow[t]{2}{*}{$\alpha_{1}$} & 0.0933 & 0.0761 & 0.0821 \\
\hline & $(0.0117)$ & $(0.0101)$ & $(0.0114)$ \\
\hline \multirow[t]{2}{*}{$\beta_{1}$} & 0.9012 & 0.9181 & 0.9127 \\
\hline & (0.0119) & $(0.0102)$ & $(0.0112)$ \\
\hline \multirow[t]{2}{*}{$v$} & 21.6701 & 13.2143 & 10.9277 \\
\hline & (7.5025) & (3.0347) & (2.2477) \\
\hline \multirow[t]{2}{*}{$\lambda$} & -0.1430 & -0.1229 & -0.1112 \\
\hline & $(0.0247)$ & $(0.0258)$ & $(0.0225)$ \\
\hline$Q(15)$ & 0.5504 & 0.5880 & 0.5372 \\
\hline$Q^{2}(15)$ & 0.2378 & 0.5044 & 0.4330 \\
\hline K-S Test & 0.8464 & 0.9835 & 0.2673 \\
\hline
\end{tabular}

Table B1. Summary statistics of the FTSE, CAC-40 and DAX daily log-returns

\begin{tabular}{lrrr}
\hline & FTSE & CAC-40 & DAX \\
\hline Mean & $1.1098 \mathrm{E}-05$ & $1.2715 \mathrm{E}-05$ & $1.3427 \mathrm{E}-04$ \\
Median & $3.3649 \mathrm{E}-04$ & $2.3667 \mathrm{E}-04$ & $8.7989 \mathrm{E}-04$ \\
Maximum & 0.0938 & 0.1059 & 0.1080 \\
Minimum & -0.0926 & -0.0947 & -0.0958 \\
Std. Deviation & 0.0131 & 0.0157 & 0.0164 \\
Asymmetry & -0.0675 & 0.0509 & -0.0048 \\
Kurtosis & 8.6249 & 7.9411 & 7.4391 \\
Jarque-Bera & $3968.9(0.000)$ & $3061.8(0.000)$ & $2470.1(0.000)$ \\
\hline
\end{tabular}

Note. Jarque-Bera corresponds to Jarque-Bera test statistics with $p$-values in parentheses.

Table B2. Estimates from the univariate ARMA-GARCH models

Note. Standard errors in parentheses. $Q(15), Q^{2}(15)$ and K-S Test are $p$-values. 
Table B3. Estimates from the AP copulas

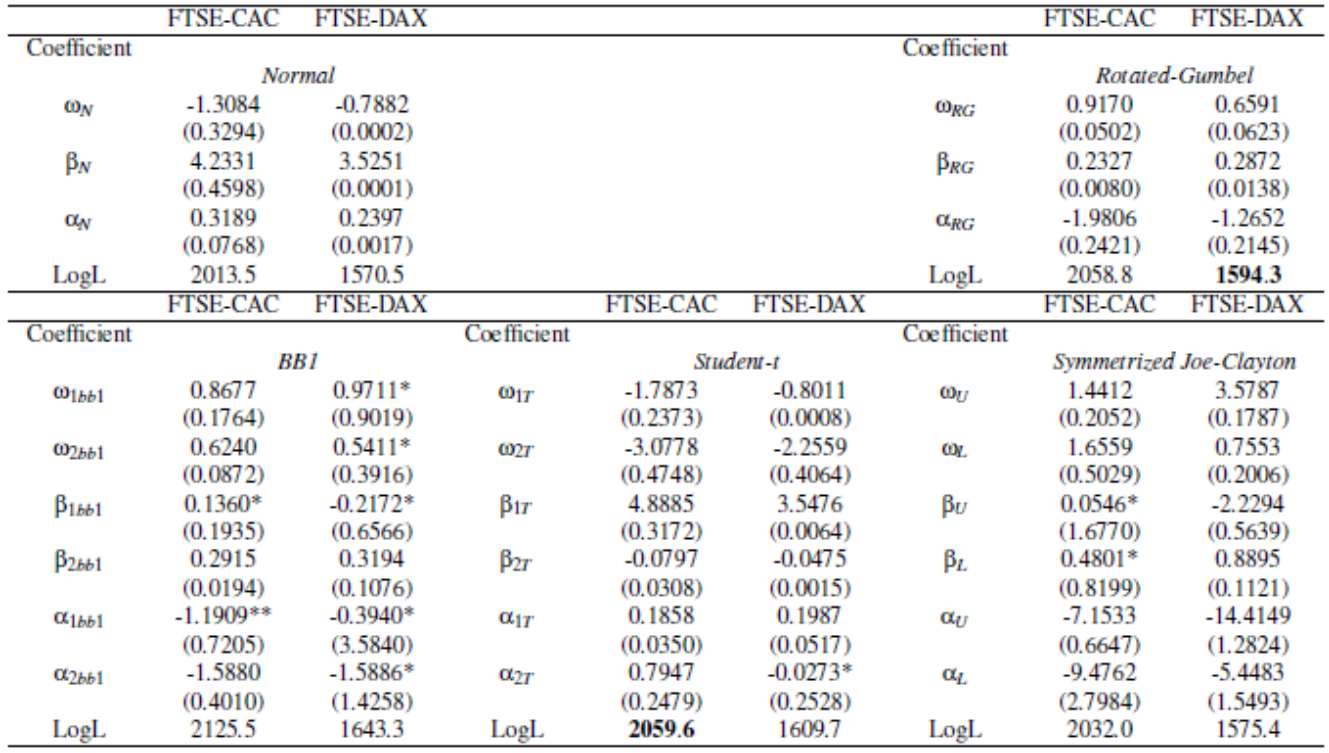

Note. Standard errors in parentheses. (*) stands for statistically not significant, whereas (**) stands for significant only at the $10 \%$ level. The notation here is as presented in Section 2 and in Appendix A.

Table B4. Estimates from the J\&R copulas

\begin{tabular}{|c|c|c|c|c|c|c|c|c|}
\hline & FTSE-CAC & FTSE-DAX & & & & & FTSE-CAC & FTSE-DAX \\
\hline \multirow[t]{2}{*}{ Coefficient } & & & & & & Coefficient & & \\
\hline & \multicolumn{2}{|c|}{ Normal } & & & & & Rotated & Gumbel \\
\hline \multirow[t]{2}{*}{$\rho_{0}$} & 0.7659 & 0.6928 & & & & $\theta_{0}$ & 2.1110 & 1.8827 \\
\hline & $(0.0186)$ & $(0.0385)$ & & & & & $(0.0938)$ & $(0.0737)$ \\
\hline \multirow[t]{2}{*}{$\rho_{1}$} & 0.9253 & 0.8988 & & & & $\theta_{1}$ & 3.7159 & 3.1997 \\
\hline & $(0.0059)$ & $(0.0153)$ & & & & & $(0.1589)$ & $(0.2039)$ \\
\hline \multirow[t]{2}{*}{$p$} & 0.9898 & 0.9785 & & & & $p$ & 0.9899 & 0.9887 \\
\hline & $(0.0733)$ & $(0.1045)$ & & & & & $(0.0771)$ & $(0.1535)$ \\
\hline \multirow[t]{2}{*}{$q$} & 0.9880 & 0.9775 & & & & $q$ & 0.9871 & 0.9886 \\
\hline & $(0.0892)$ & $(0.1431)$ & & & & & $(0.0839)$ & $(0.1358)$ \\
\hline \multirow[t]{2}{*}{$\log \mathrm{L}$} & 2128.8 & 1666.0 & & & & LogL & 2045.0 & 1601.2 \\
\hline & FTSE-CAC & FTSE-DAX & & FTSE-CAC & FTSE-DAX & & FTSE-CAC & FTSE-DAX \\
\hline \multirow[t]{2}{*}{ Coefficient } & & & Coefficient & & & Coefficient & & \\
\hline & \multicolumn{2}{|c|}{$B B 1$} & & \multicolumn{2}{|c|}{ Student-t $t$} & & \multicolumn{2}{|c|}{ Symmetrized Joe-Clayton } \\
\hline \multirow[t]{2}{*}{$\kappa_{0}$} & 0.5547 & 0.4812 & $\rho_{0}$ & 0.7793 & 0.7093 & $\lambda_{v 0}$ & 0.5434 & 0.4673 \\
\hline & $(0.1038)$ & $(0.0837)$ & & $(0.0192)$ & $(0.0252)$ & & $(0.0278)$ & $(0.0381)$ \\
\hline \multirow[t]{2}{*}{$\kappa_{1}$} & 0.8168 & 0.8197 & $\rho_{1}$ & 0.9275 & 0.8818 & $\lambda_{U 1}$ & 0.8011 & 0.6556 \\
\hline & $(0.1223)$ & $(0.1378)$ & & $(0.0076)$ & $(0.0194)$ & & $(0.0000)$ & $(0.0054)$ \\
\hline$\gamma_{0}$ & $\begin{array}{c}1.7324 \\
(0.0819)\end{array}$ & $\begin{array}{c}1.5566 \\
(0.0718)\end{array}$ & $v_{0}$ & $\begin{array}{l}11.5834 \\
(3.6496)\end{array}$ & $\begin{array}{l}13.4763 \\
(6.1923)\end{array}$ & $\lambda_{L 0}$ & $\begin{array}{c}0.6167 \\
(0.0178)\end{array}$ & $\begin{array}{c}0.5031 \\
(0.0425)\end{array}$ \\
\hline$\gamma_{1}$ & 2.6850 & 2.1590 & $\mathrm{v}_{1}$ & $41.3768^{*}$ & 14.5128 & $\lambda_{L 1.1}$ & 0.7804 & 0.7539 \\
\hline & $(0.2337)$ & $(0.0862)$ & & $(54.9352)$ & $(6.8262)$ & & $(0.0001)$ & $(0.0743)$ \\
\hline$p$ & 0.9910 & 0.9953 & $p$ & 0.9906 & $0.9963^{* *}$ & $p$ & 0.9868 & 0.9931 \\
\hline & $(0.0736)$ & $(0.2303)$ & & $(0.0827)$ & $(0.5380)$ & & $(0.0796)$ & $(0.1811)$ \\
\hline$q$ & 0.9900 & 0.9948 & $q$ & 0.9906 & $0.9959^{* *}$ & $q$ & 0.9883 & 0.9924 \\
\hline & $(0.0837)$ & $(0.2269)$ & & $(0.0814)$ & $(0.5286)$ & & $(0.0984)$ & $(0.1945)$ \\
\hline LogL & 2102.8 & 1637.4 & $\log \mathrm{L}$ & 2138.1 & 1671.7 & LogL & 2003.8 & 1570.0 \\
\hline
\end{tabular}

Note. Standard errors in parentheses. $(*)$ stands for statistically not significant, whereas (**) stands for significant only at the $10 \%$ level. The notation here is as presented in Section 2 and in Appendix A. 
Table B5. Estimates from the SFZ\&D copulas

\begin{tabular}{|c|c|c|c|c|c|c|c|c|}
\hline & FTSE-CAC & FTSE-DAX & & & & & FTSE-CAC & FTSE-DAX \\
\hline \multirow[t]{2}{*}{ Coefficient } & & & & & & \multicolumn{3}{|l|}{ Coefficient } \\
\hline & \multicolumn{2}{|c|}{ Normal } & & & & \multirow[b]{2}{*}{$\omega_{n C D}$} & \multicolumn{2}{|c|}{ Rosared-Gumbel } \\
\hline$\omega_{v} 0$ & $\begin{array}{l}0.4226^{*} \\
(0.6034)\end{array}$ & $\begin{array}{c}1.3001 \\
(0.1364)\end{array}$ & & & & & $\begin{array}{l}-1.0715 \\
(0.0916)\end{array}$ & $\begin{array}{c}-0.4675 \\
(0.0867)\end{array}$ \\
\hline$\omega_{\mathrm{N} 1}$ & $\begin{array}{l}1.2766^{* * *} \\
(0.7531)\end{array}$ & $\begin{array}{c}2.3473 \\
(0.1020)\end{array}$ & & & & $\omega_{R G 1}$ & $\begin{array}{c}0.9764 \\
(0.0538)\end{array}$ & $\begin{array}{c}2.5377 \\
(0.0740)\end{array}$ \\
\hline \multirow[t]{2}{*}{$\beta_{N}$} & $1.7286^{* * *}$ & 0.1549 & & & & \multirow[t]{2}{*}{$\beta_{K G G}$} & 0.2222 & -0.3209 \\
\hline & $(0.9405)$ & $(0.0657)$ & & & & & $(0.0081)$ & $(0.0142)$ \\
\hline \multirow[t]{2}{*}{$\alpha_{N}$} & 0.3493 & 0.3737 & & & & \multirow[t]{2}{*}{$\alpha_{R G}$} & -2.0588 & $0.6656^{* *}$ \\
\hline & $(0.1084)$ & $(0.0921)$ & & & & & $(0.2819)$ & $(0.3521)$ \\
\hline$p$ & $\begin{array}{c}0.9922 \\
(0.0826)\end{array}$ & $\begin{array}{c}0.9900 \\
(0.2225)\end{array}$ & & & & $p$ & $\begin{array}{c}0.9978 \\
(0.0794)\end{array}$ & $\begin{array}{c}0.9910 \\
(0.1584)\end{array}$ \\
\hline \multirow[t]{2}{*}{$q$} & 0.9888 & 0.9864 & & & & \multirow[t]{2}{*}{$q$} & 0.9894 & 0.9945 \\
\hline & $(0.1076)$ & $(0.2934)$ & & & & & $(0.2109)$ & $(0.1605)$ \\
\hline \multirow[t]{2}{*}{$\log \mathrm{L}$} & 2145.1 & 1675.2 & & & & \multirow[t]{2}{*}{$\log \mathrm{L}$} & 2079.7 & 1604.2 \\
\hline & FTSE-CAC & FTSE-DAX & & FTSE-CAC & FTSE-DAX & & FTSE-CAC & FTSE-DAX \\
\hline \multirow[t]{2}{*}{ Coefficient } & \multirow{2}{*}{\multicolumn{2}{|c|}{$B B I$}} & Coefficient & \multirow{2}{*}{\multicolumn{2}{|c|}{ Srudent-t }} & \multirow[t]{2}{*}{ Coefficient } & \multirow{2}{*}{\multicolumn{2}{|c|}{ Symmerrized Joe-Clayton }} \\
\hline & & & & & & & & \\
\hline$\omega_{10 b b 1}$ & $\begin{array}{l}0.2880^{* *} \\
(0.1668)\end{array}$ & $\begin{array}{r}-0.4544 \\
0.2262)\end{array}$ & $\omega_{10 r}$ & $\begin{array}{l}-0.6644 \\
(0.6588)\end{array}$ & $\begin{array}{r}-0.0229 \\
00.0085\end{array}$ & $\omega_{20}$ & 1.1802 & $\begin{array}{l}2.1219 \\
0.1156\end{array}$ \\
\hline \multirow[t]{2}{*}{$\omega_{11 b b 1}$} & 0.5221 & 0.9416 & $\omega_{11 T}$ & $-0.0393^{* * *}$ & 0.5144 & \multirow[t]{2}{*}{$\omega_{v 1}$} & $\begin{array}{l}(0.108 /) \\
1.7125\end{array}$ & 2.1413 \\
\hline & $(0.0482)$ & $(0.2045)$ & & $(0.0236)$ & $(0.0526)$ & & $(0.0045)$ & $(0.2027)$ \\
\hline$\omega_{20 b b 1}$ & -1.4688 & -0.4549 & vo & 11.9186 & 12.1589 & $\omega_{10}$ & 0.1471 & $-0.0631^{*}$ \\
\hline & $(0.2986)$ & $(0.1928)$ & & $(3.7720)$ & $(4.0464)$ & & $(0.0783)$ & $(0.1197)$ \\
\hline$\omega_{21 b b 1}$ & $\begin{array}{c}0.7128 \\
(0.0663)\end{array}$ & $\begin{array}{c}1.5930 \\
(0.2089)\end{array}$ & $\mathrm{v}_{1}$ & $\begin{array}{l}15.0000 \\
(2.4045)\end{array}$ & $\begin{array}{l}15.0000 \\
(3.7565)\end{array}$ & $\omega_{L, 1}$ & $\begin{array}{c}0.9387 \\
(0.0019)\end{array}$ & $\begin{array}{c}0.6713 \\
(0.0682)\end{array}$ \\
\hline$\beta_{1 b b 1}$ & 0.4861 & 0.2808 & $\beta_{1 T}$ & 3.3971 & 2.3508 & $\beta_{v}$ & -0.2633 & -0.6172 \\
\hline & $(0.0404)$ & $(0.0849)$ & & $(0.1194)$ & $(0.0561)$ & & $(0.0001)$ & $(0.1901)$ \\
\hline$\beta_{2 b b 1}$ & 0.2714 & -0.3478 & $\alpha_{1 T}$ & 0.1114 & 0.1492 & $\beta_{L}$ & 0.6745 & 1.0390 \\
\hline & $(0.0153)$ & $(0.0609)$ & & $(0.0271)$ & $(0.0351)$ & & $(0.0008)$ & $(0.0480)$ \\
\hline$\alpha_{1 b b 1}$ & $-0.1265^{*}$ & -2.1677 & $\cdots$ & $\ldots$ & $\ldots$ & $\alpha_{v}$ & -5.4357 & -10.7196 \\
\hline & $(0.1232)$ & $(0.9771)$ & & ... & $\cdots$ & & $(0.0214)$ & $(0.6474)$ \\
\hline$\alpha_{2 b_{1} 1}$ & -2.1346 & 1.4201 & ... & ... & $\cdots$ & $\alpha_{L}$ & -1.6781 & -2.7932 \\
\hline & $(0.3201)$ & $(0.5684)$ & & $\ldots$ & $\ldots$ & & $(0.0050)$ & $(0.0344)$ \\
\hline$p$ & 0.9965 & 0.9952 & $p$ & 0.9922 & 0.9982 & $p$ & 0.9946 & 0.9931 \\
\hline & $(0.1681)$ & $(0.2316)$ & & $(0.0793)$ & $(0.2217)$ & & $(0.0614)$ & $(0.1428)$ \\
\hline$q$ & 0.9780 & 0.9957 & $q$ & 0.9918 & 0.9980 & $q$ & 0.9914 & 0.9923 \\
\hline & $(0.3329)$ & $(0.1968)$ & & $(0.0764)$ & $(0.2067)$ & & $(0.0794)$ & $(0.1647)$ \\
\hline $\log \mathrm{L}$ & 2143.2 & 1657.2 & $\log \mathrm{L}$ & 2147.4 & 1683.8 & $\log \mathrm{L}$ & 2037.4 & 1599.2 \\
\hline
\end{tabular}

Note. Standard errors in parentheses. (*) stands for statistically not significant, whereas (**) stands for significant only at the $10 \%$ level. The notation here is as presented in Section 2 and in Appendix A.

Table B6. Estimates from the TZ\&SF copulas

\begin{tabular}{|c|c|c|c|c|c|c|c|c|}
\hline & FTSE-CAC & FTSE-DAX & & FTSE-CAC & FTSE-DAX & & FTSE-CAC & FTSE-DAX \\
\hline \multirow[t]{2}{*}{ Coefficient } & & & Coefficient & \multirow{2}{*}{\multicolumn{2}{|c|}{ NormaVGumbel }} & Coefficient & & \\
\hline & \multicolumn{2}{|c|}{ NormaVClayton } & & & & & \multicolumn{2}{|c|}{ NormaVRorased-Gumbel } \\
\hline \multirow[t]{2}{*}{$\omega_{N}$} & -3.4314 & $1.5731^{*}$ & $\omega_{v}$ & -3.4070 & 5.1152 & $\omega_{\mathrm{v}}$ & -3.6888 & 0.7360 \\
\hline & $(0.1346)$ & $(8.5125)$ & & $(0.3598)$ & $(0.2004)$ & & $(0.1479)$ & $(0.0223)$ \\
\hline$\omega_{C}$ & $\begin{array}{c}-0.5596 \\
(0.0705)\end{array}$ & $\begin{array}{l}-1.0128 \\
(0.3585)\end{array}$ & $\omega_{G}$ & $\begin{array}{l}1.0634^{*} \\
(5.54511\end{array}$ & $\begin{array}{l}0.3575^{*} \\
(0.3557)\end{array}$ & $\omega_{R G}$ & $\begin{array}{l}1.0596 \\
(0.2003)\end{array}$ & $\begin{array}{l}-1.6510 \\
0.15100\end{array}$ \\
\hline \multirow[t]{2}{*}{$\beta_{N}$} & 6.9297 & $0.9382^{*}$ & $\beta_{\mathrm{N}}$ & 6.8881 & -3.1589 & $\beta_{N}$ & 7.2237 & 1.9599 \\
\hline & $(0.1616)$ & $(9.9745)$ & & $(0.4349)$ & $(0.2944)$ & & $(0.1739)$ & $(0.0391)$ \\
\hline \multirow[t]{2}{*}{$\beta_{C}$} & -0.4039 & 0.4156 & $\beta_{G}$ & $0.0730^{*}$ & 0.2976 & $\beta_{R G}$ & $0.0524^{*}$ & 0.4867 \\
\hline & $(0.0290)$ & $(0.0426)$ & & (2.1589) & $(0.0858)$ & & $(0.0948)$ & $(0.0431)$ \\
\hline$\alpha_{N}$ & $\begin{array}{c}0.1779 \\
(0.0252)\end{array}$ & $\begin{array}{c}0.2852^{* * *} \\
(0.1540)\end{array}$ & $\alpha_{N}$ & $\begin{array}{c}0.2403 \\
(0.0482)\end{array}$ & $\begin{array}{c}0.3732^{* * *} \\
(0.1919)\end{array}$ & $\alpha_{N}$ & $\begin{array}{c}0.2256 \\
(0.0300)\end{array}$ & $\begin{array}{c}0.2995 \\
(0.0598)\end{array}$ \\
\hline \multirow{2}{*}{$\alpha_{C}$} & -0.2491 & $-1.8673^{*}$ & $\alpha_{G}$ & $-1.3699^{*}$ & $-0.2412^{*}$ & $\alpha_{R G}$ & $-0.9710^{*}$ & $-0.3152^{*}$ \\
\hline & $(0.1009)$ & (1.5211) & & (7.7933) & $(0.9439)$ & & $(0.7403)$ & $(0.7471)$ \\
\hline \multirow[t]{2}{*}{$p$} & 0.9898 & 0.9856 & $p$ & 0.9944 & 0.9891 & $p$ & 0.9937 & 0.9900 \\
\hline & $(0.0988)$ & $(0.0889)$ & & $(0.0635)$ & $(0.0915)$ & & $(0.0930)$ & $(0.0655)$ \\
\hline$q$ & 0.9512 & 0.9474 & $q$ & 0.9876 & 0.9740 & $q$ & 0.9875 & 0.9793 \\
\hline \multirow{2}{*}{$\log L$} & $\begin{array}{c}(0.2973) \\
2095.7\end{array}$ & (0.1948) & I & $\begin{array}{l}(0.1012) \\
21109\end{array}$ & $\begin{array}{l}(0.2020) \\
16347\end{array}$ & LogL & $(0.1095)$ & $\begin{array}{c}(0.1144) \\
16509\end{array}$ \\
\hline & FTSE-CAC & FTSE-DAX & Dogh & 2119.9 & 1034.7 & Wogh & $\begin{array}{l}2130.9 \\
\text { FTSE-CAC }\end{array}$ & FTSE-DAX \\
\hline \multirow[t]{2}{*}{ Coefficient } & \multirow{2}{*}{\multicolumn{2}{|c|}{ NormaVBBI }} & & & & Coefficient & & \\
\hline & & & & & & & \multicolumn{2}{|c|}{ NormaVSymmerrized Joe-Clayson } \\
\hline \multirow[t]{2}{*}{$\omega_{N}$} & -3.9135 & $1.6258^{* *}$ & & & & $\omega_{\mathrm{N}}$ & -3.4995 & 1.4217 \\
\hline & $(0.1430)$ & $(0.8553)$ & & & & & (1.0684) & $(0.4894)$ \\
\hline \multirow[t]{2}{*}{$\omega_{1 b b 1}$} & 0.4021 & 1.2382 & & & & $\omega_{t y}$ & $-0.0842^{*}$ & 0.2897 \\
\hline & $(0.0438)$ & $(0.4464)$ & & & & & (3.1934) & $(0.1237)$ \\
\hline \multirow[t]{2}{*}{$\omega_{2 b b 1}$} & 0.8370 & $0.3780^{*}$ & & & & $\omega_{\mathrm{E}}$ & $0.5731^{*}$ & 1.4937 \\
\hline & $(0.4011)$ & $(0.6279)$ & & & & & $(4.2548)$ & $(0.0061)$ \\
\hline \multirow[t]{2}{*}{$\beta_{N}$} & 7.4839 & $-0.3579^{*}$ & & & & $\beta_{N}$ & 6.9979 & $0.1849^{*}$ \\
\hline & $(0.1666)$ & $(1.4047)$ & & & & & (1.2537) & $(0.5423)$ \\
\hline \multirow[t]{2}{*}{$\beta_{1 b b 1}$} & 0.5597 & $-0.1331^{*}$ & & & & $\beta v$ & $1.3566^{*}$ & 1.9657 \\
\hline & $(0.0597)$ & $(0.2992)$ & & & & & $(8.2579)$ & $(0.0392)$ \\
\hline \multirow[t]{2}{*}{$\beta_{2 b b 1}$} & $0.1587^{*}$ & $0.3600^{* *}$ & & & & $\beta_{2}$ & $-0.1108^{*}$ & -0.0790 \\
\hline & $(0.1678)$ & $(0.1857)$ & & & & & $(0.2036)$ & $(0.0258)$ \\
\hline \multirow[t]{2}{*}{$\alpha_{N}$} & 0.2413 & 0.5031 & & & & $\alpha_{N}$ & 0.2389 & 0.4739 \\
\hline & $(0.0348)$ & $(0.1864)$ & & & & & $(0.0619)$ & $(0.2082)$ \\
\hline$\alpha_{1 b b 1}$ & $0.1784^{*}$ & $-1.7946^{*}$ & & & & $\alpha_{U}$ & $-3.6760^{*}$ & -8.0732 \\
\hline & $(0.2448)$ & $(4.6244)$ & & & & & (12.0692) & (1.8708) \\
\hline$\alpha_{2 b b 1}$ & -1.6811 & $-0.7594^{*}$ & & & & $\alpha_{L}$ & $\begin{array}{l}-1.7287^{*} \\
(6.5903\end{array}$ & $\begin{array}{l}-1.8739 \\
-1.0807\end{array}$ \\
\hline & $(0.7918)$ & $(2.1270)$ & & & & & (6.5093) & $(0.0897)$ \\
\hline$p$ & 0.9945 & 0.9859 & & & & $p$ & 0.9945 & 0.9822 \\
\hline & $(0.0805)$ & $(0.2587)$ & & & & & $(0.1822)$ & $(0.0598)$ \\
\hline$q$ & 0.9923 & 0.9894 & & & & $q$ & 0.9887 & 0.9757 \\
\hline & $(0.1263)$ & $(0.2219)$ & & & & $\pi$ & $(0.1320)$ & $(0.0384)$ \\
\hline $\log \mathrm{L}$ & 2151.8 & 1667.4 & & & & $\log \mathrm{L}$ & 2137.0 & 1633.5 \\
\hline
\end{tabular}


Table B7. Results of the Kupiec and Christoffersen tests for the daily VaR forecasts

\begin{tabular}{|c|c|c|c|c|c|c|c|c|c|c|c|c|}
\hline \multirow[b]{3}{*}{ Copula } & \multirow{2}{*}{\multicolumn{5}{|c|}{ AP copulas }} & \multicolumn{3}{|c|}{ FTSE-CAC } & \multirow{2}{*}{\multicolumn{4}{|c|}{ SFZ\&D copulas }} \\
\hline & & & & & & & $J \& R \operatorname{cog}$ & ulas & & & & \\
\hline & $\alpha$ & $\pi_{o b s}$ & K & C & $\alpha$ & $\pi_{o b s}$ & $\mathrm{~K}$ & $\mathrm{C}$ & $\alpha$ & $\pi_{o b s}$ & $\mathrm{~K}$ & $\mathrm{C}$ \\
\hline \multirow[t]{2}{*}{ Normal } & $1 \%$ & 0.0093 & 0.6931 & 0.7047 & $1 \%$ & 0.0086 & 0.4387 & 0.5857 & $\ldots$ & $\ldots$ & $\ldots$ & $\ldots$ \\
\hline & $5 \%$ & 0.0501 & 0.9767 & 0.9891 & $5 \%$ & 0.0514 & 0.7174 & 0.8280 & & & & \\
\hline \multirow[t]{2}{*}{ Student-t } & $1 \%$ & 0.0096 & 0.8351 & 0.7310 & $\ldots$ & $\ldots$ & $\ldots$ & $\ldots$ & $1 \%$ & 0.0090 & 0.5596 & 0.4225 \\
\hline & $5 \%$ & 0.0531 & 0.4389 & 0.5157 & & & & & $5 \%$ & 0.0561 & 0.1321 & 0.2991 \\
\hline \multirow[t]{2}{*}{ BB1 } & $\ldots$ & $\ldots$ & $\ldots$ & $\ldots$ & $1 \%$ & 0.0056 & 0.0088 & 0.0293 & $\ldots$ & $\ldots$ & $\ldots$ & $\ldots$ \\
\hline & $\ldots$ & $\ldots$ & $\ldots$ & $\ldots$ & $5 \%$ & 0.0451 & 0.2134 & 0.3892 & & & & \\
\hline \multirow[t]{2}{*}{ SJC } & $\ldots$ & $\ldots$ & $\ldots$ & $\ldots$ & $1 \%$ & 0.0083 & 0.3332 & 0.5037 & $1 \%$ & 0.0083 & 0.3332 & 0.5037 \\
\hline & & & & & $5 \%$ & 0.0531 & 0.4389 & 0.6097 & $5 \%$ & 0.0538 & 0.3483 & 0.5506 \\
\hline \multirow[t]{4}{*}{ Rotated-Gumbel } & $1 \%$ & 0.0080 & 0.2446 & 0.4157 & $1 \%$ & 0.0090 & 0.5596 & 0.6548 & $1 \%$ & 0.0080 & 0.2446 & 0.4157 \\
\hline & $5 \%$ & 0.0475 & 0.5191 & 0.7318 & $5 \%$ & 0.0498 & 0.9566 & 0.9297 & $5 \%$ & 0.0485 & 0.6961 & 0.4849 \\
\hline & \multicolumn{12}{|c|}{ FTSE-DAX } \\
\hline & & & AP copu & & & & $J \& R \operatorname{cog}$ & ulas & & SFZ & \&D copul & \\
\hline \multirow{3}{*}{$\frac{\text { Copula }}{\text { Normal }}$} & $\alpha$ & $\pi_{o b s}$ & K & $\mathrm{C}$ & $\alpha$ & $\pi_{o b s}$ & $\mathrm{~K}$ & $\mathrm{C}$ & $\alpha$ & $\pi_{o b s}$ & K & $\mathrm{C}$ \\
\hline & $1 \%$ & 0.0093 & 0.6931 & 0.7047 & $1 \%$ & 0.0090 & 0.5596 & 0.4225 & $1 \%$ & 0.0086 & 0.4387 & 0.3497 \\
\hline & $5 \%$ & 0.0524 & 0.5420 & 0.8240 & $5 \%$ & 0.0518 & 0.6565 & 0.6772 & $5 \%$ & 0.0508 & 0.8446 & 0.8400 \\
\hline \multirow[t]{2}{*}{ Student-t } & $\ldots$ & $\ldots$ & $\ldots$ & $\ldots$ & $1 \%$ & 0.0076 & 0.1731 & 0.3287 & $1 \%$ & 0.0103 & 0.8740 & 0.6067 \\
\hline & & & & & $5 \%$ & 0.0528 & 0.4889 & 0.4923 & $5 \%$ & 0.0524 & 0.5420 & 0.7824 \\
\hline \multirow[t]{2}{*}{ BB1 } & $\ldots$ & $\ldots$ & $\ldots$ & $\ldots$ & $1 \%$ & 0.0043 & 0.0004 & 0.0018 & $1 \%$ & 0.0043 & 0.0004 & 0.0018 \\
\hline & $\ldots$ & & & & $5 \%$ & 0.0465 & 0.3679 & 0.5328 & $5 \%$ & 0.0455 & 0.2469 & 0.4643 \\
\hline \multirow[t]{2}{*}{ SJC } & $1 \%$ & 0.0090 & 0.5596 & 0.6548 & $1 \%$ & 0.0093 & 0.6931 & 0.4899 & $\ldots$ & $\ldots$ & $\ldots$ & $\ldots$ \\
\hline & $5 \%$ & 0.0521 & 0.5980 & 0.8229 & $5 \%$ & 0.0511 & 0.7802 & 0.8893 & $\ldots$ & $\ldots$ & $\ldots$ & $\ldots$ \\
\hline \multirow[t]{2}{*}{ Rotated-Gumbel } & $1 \%$ & 0.0083 & 0.3332 & 0.2887 & $1 \%$ & 0.0086 & 0.4387 & 0.3497 & $1 \%$ & 0.0083 & 0.3332 & 0.2774 \\
\hline & $5 \%$ & 0.0504 & 0.9103 & 0.9881 & $5 \%$ & 0.0498 & 0.9566 & 0.8054 & $5 \%$ & 0.0495 & 0.8901 & 0.7806 \\
\hline
\end{tabular}

Note. $\pi_{o b s}$ is the observed proportion of violations. $\mathrm{K}$ and $\mathrm{C}$ correspond to the $p$-values of the Kupiec and Christoffersen tests, respectively.

Table B8. Average losses computed based on the $1 \%$ daily VaR forecasts and the results of the SPA test

\begin{tabular}{|c|c|c|c|}
\hline Benchmark & $\begin{array}{r}\text { AP copulas } \\
\text { Average Loss }(\%)\end{array}$ & $\begin{array}{c}\text { FTSE-CAC } \\
\text { J\&R copulas } \\
\text { Average Loss }(\%) \\
\end{array}$ & $\begin{array}{c}\text { SFZ\&D copulas } \\
\text { Average Loss }(\%)\end{array}$ \\
\hline Normal & $\begin{array}{c}9.7856 \\
(1.0000)\end{array}$ & $\begin{array}{c}9.7691 \\
(1.0000)\end{array}$ & $\ldots$ \\
\hline Student-t & $\begin{array}{l}9.9677 \\
(0.0000)\end{array}$ & $\ldots$ & $\begin{array}{c}9.8933 \\
(1.0000)\end{array}$ \\
\hline SJC & $\ldots$ & $\begin{array}{c}9.9931 \\
(0.0000)\end{array}$ & $\begin{array}{c}9.9952 \\
(0.0012)\end{array}$ \\
\hline Rotated-Gumbel & $\begin{array}{l}10.0320 \\
(0.0000)\end{array}$ & $\begin{array}{l}9.9397 \\
(0.0000)\end{array}$ & $\begin{array}{c}9.9747 \\
(0.0001)\end{array}$ \\
\hline Benchmark & $\begin{array}{r}\text { AP copulas } \\
\text { Average Loss }(\%)\end{array}$ & $\begin{array}{l}\text { FTSE-DAX } \\
\text { J\&R copulas } \\
\text { Average Loss }(\%)\end{array}$ & $\begin{array}{l}\text { SFZ\&D copulas } \\
\text { Average Loss }(\%)\end{array}$ \\
\hline Normal & $\begin{array}{c}9.9492 \\
(1.0000)\end{array}$ & $\begin{array}{c}9.7691 \\
(1.0000)\end{array}$ & $\begin{array}{c}9.9681 \\
(1.0000)\end{array}$ \\
\hline Student-t & $\ldots$ & $\begin{array}{l}9.9250 \\
(0.0002)\end{array}$ & $\begin{array}{l}10.0754 \\
(0.0010)\end{array}$ \\
\hline SJC & $\begin{array}{l}10.2461 \\
(0.0000)\end{array}$ & $\begin{array}{l}10.3284 \\
(0.0000)\end{array}$ & $\ldots$ \\
\hline Rotated-Gumbel & $\begin{array}{l}10.2728 \\
(0.0000)\end{array}$ & $\begin{array}{l}10.1896 \\
(0.0000)\end{array}$ & $\begin{array}{l}10.2184 \\
(0.0000)\end{array}$ \\
\hline
\end{tabular}

Note. In parentheses, we have the $p$-value of the SPA test.

Table B9. Average losses computed based on the 1\% daily VaR forecasts and the results of the SPA test for the best models out of each methodology for the pairs FTSE-CAC and FTSE-DAX

\begin{tabular}{cccc}
\hline FTSE-CAC & & \multicolumn{2}{c}{ FTSE-DAX } \\
Benchmark & Average Loss $(\%)$ & Benchmark & Average Loss $(\%)$ \\
\hline Normal (AP copula) & 9.7856 & Normal (AP copula) & 9.9493 \\
& $(0.0339)$ & & $(\mathbf{1 . 0 0 0 0})$ \\
Normal (J\&R copula) & $\mathbf{9 . 7 6 9 1}$ & Normal (J\&R copula) & 9.9814 \\
& $(\mathbf{1 . 0 0 0 0})$ & & $(0.0000)$ \\
Student-t (SFZ\&D copula) & 9.8933 & Normal (SFZ\&D copula) & 9.9681 \\
& $(0.0000)$ & & $(0.0338)$ \\
Normal-Clayton (TZ\&SF copula) & 9.9922 & $\ldots$ & $\ldots$ \\
& $(0.0000)$ & & $\ldots$ \\
\hline
\end{tabular}

Note. In parentheses, we have the $p$-value of the SPA test. 


\section{Appendix C}

(a) Student-t
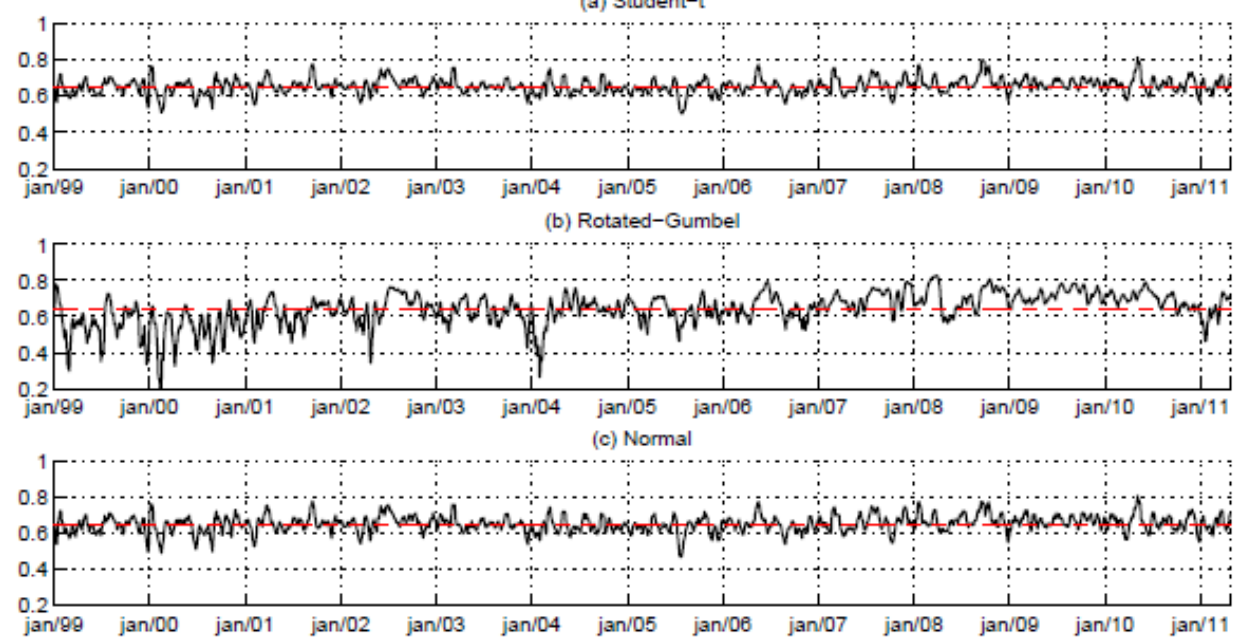

Figure C1. The evolution of the Kendall's tau computed based on the AP copulas, for the FTSE-CAC pair

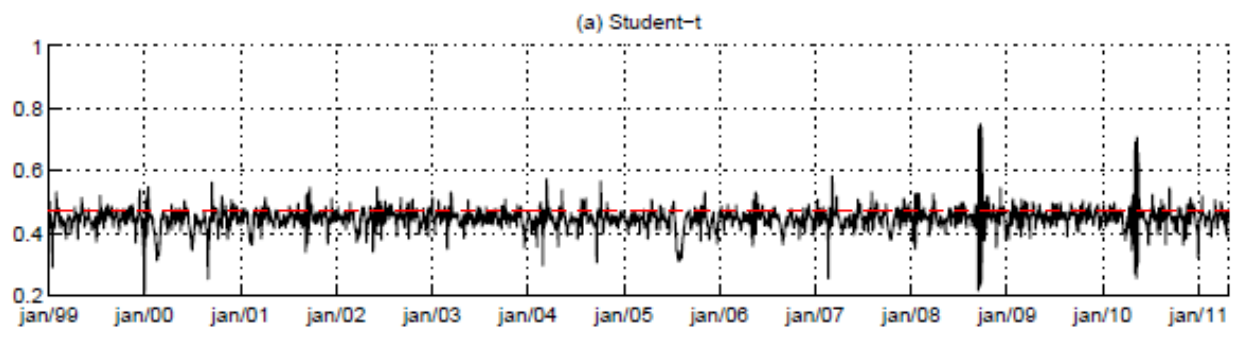

(b) Rotated-Gumbel

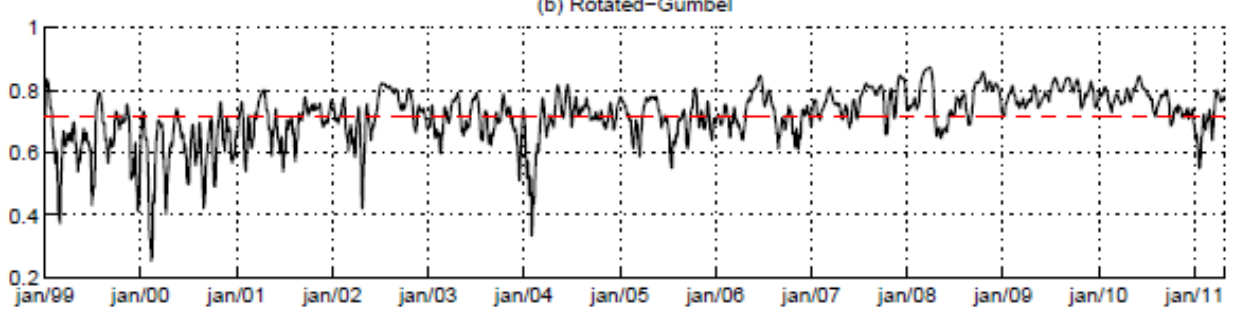

Figure $\mathrm{C} 2$. The evolutions of the tail dependence parameters computed based on the AP copulas, for the FTSE-CAC pair

(a) Rotated-Gumbe
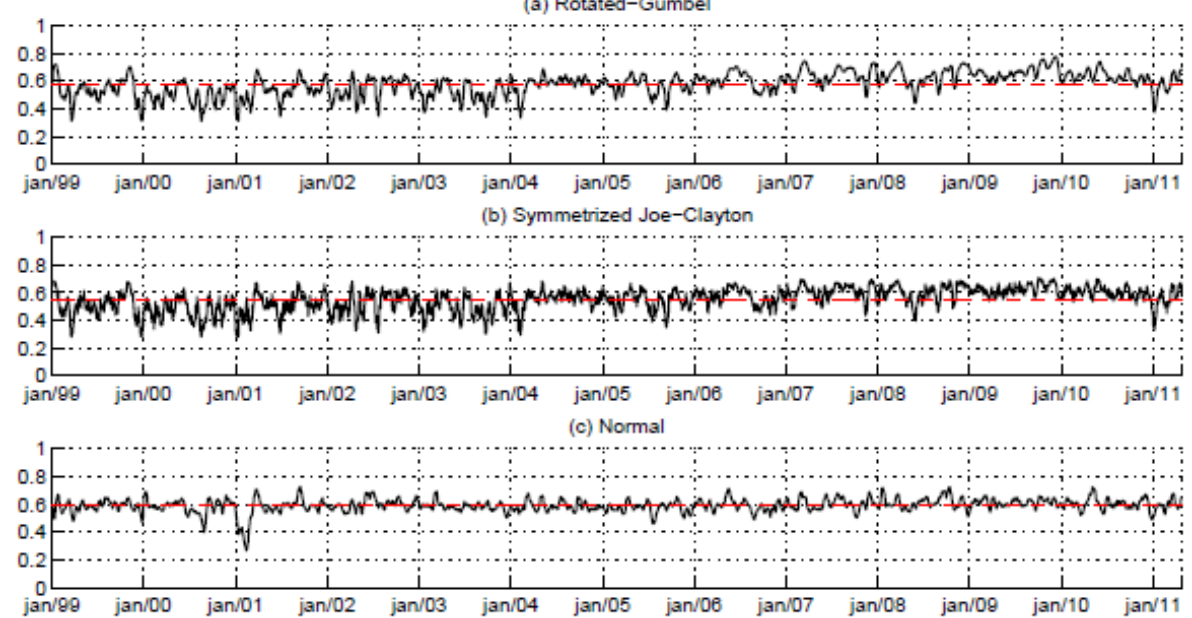

Figure C3. The evolution of the Kendall's tau computed based on the AP copulas, for the FTSE-DAX pair 

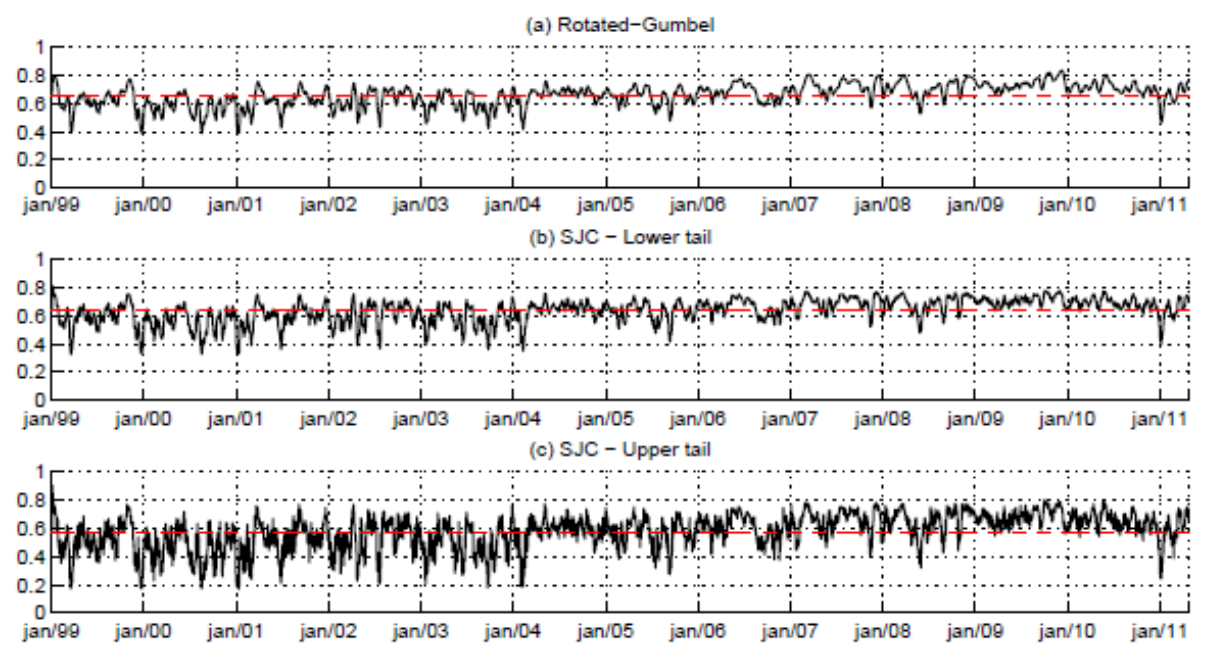

Figure $\mathrm{C} 4$. The evolutions of the tail dependence parameters computed based on the AP copulas, for the FTSE-DAX pair

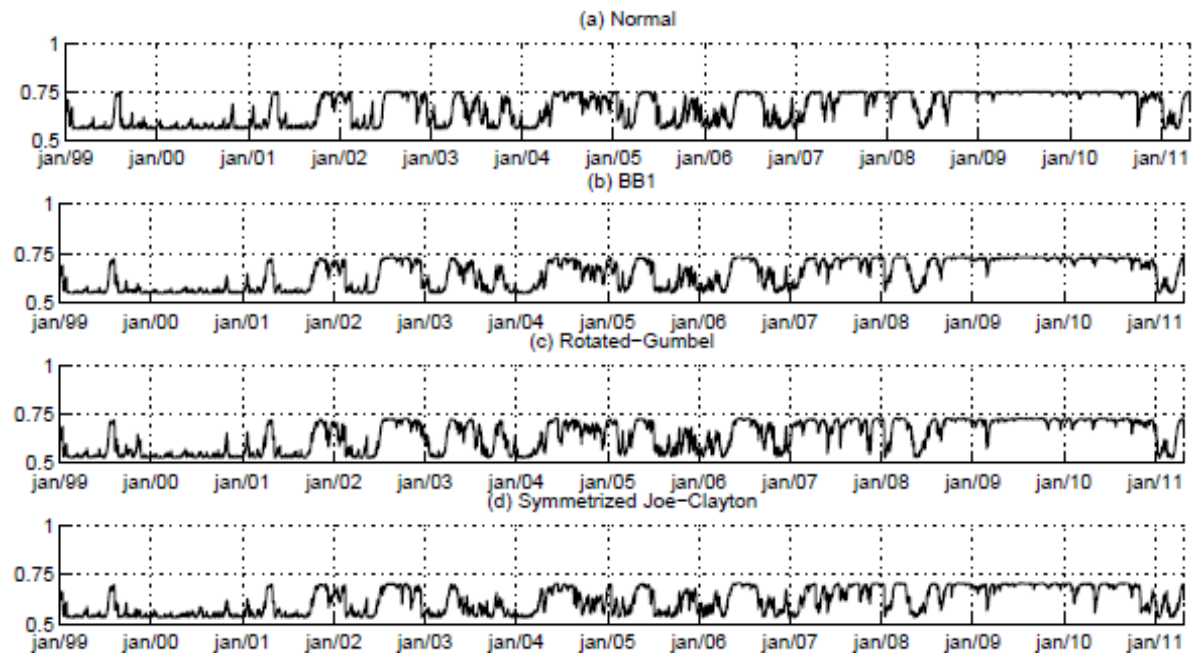

Figure C5. The evolution of the Kendall's tau computed based on the J\&R copulas, for the FTSE-CAC pair

(a) BB1

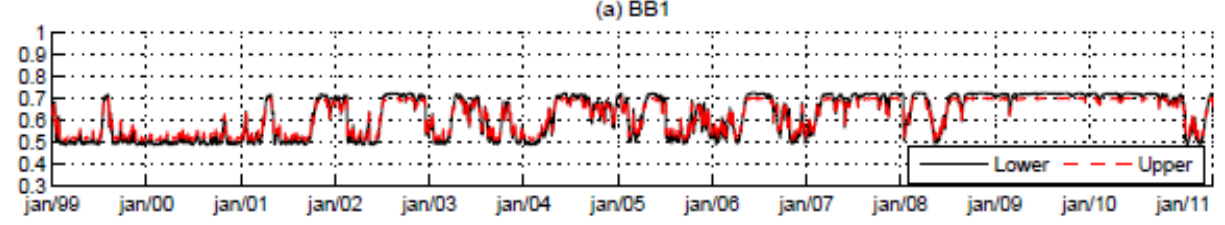

(b) Rotated-Gumbel

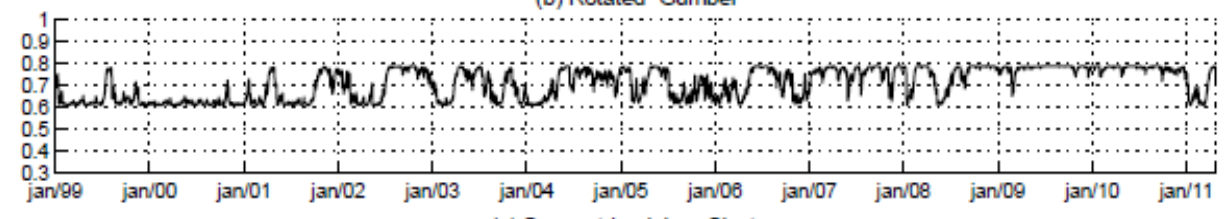

(c) Symmetrized Joe-Clayton

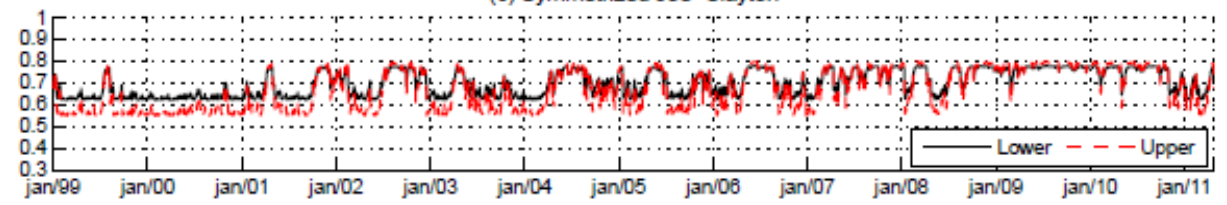

Figure C6. The evolutions of the tail dependence parameters computed based on the J\&R copulas, for the FTSE-CAC pair 
(a) Student-t

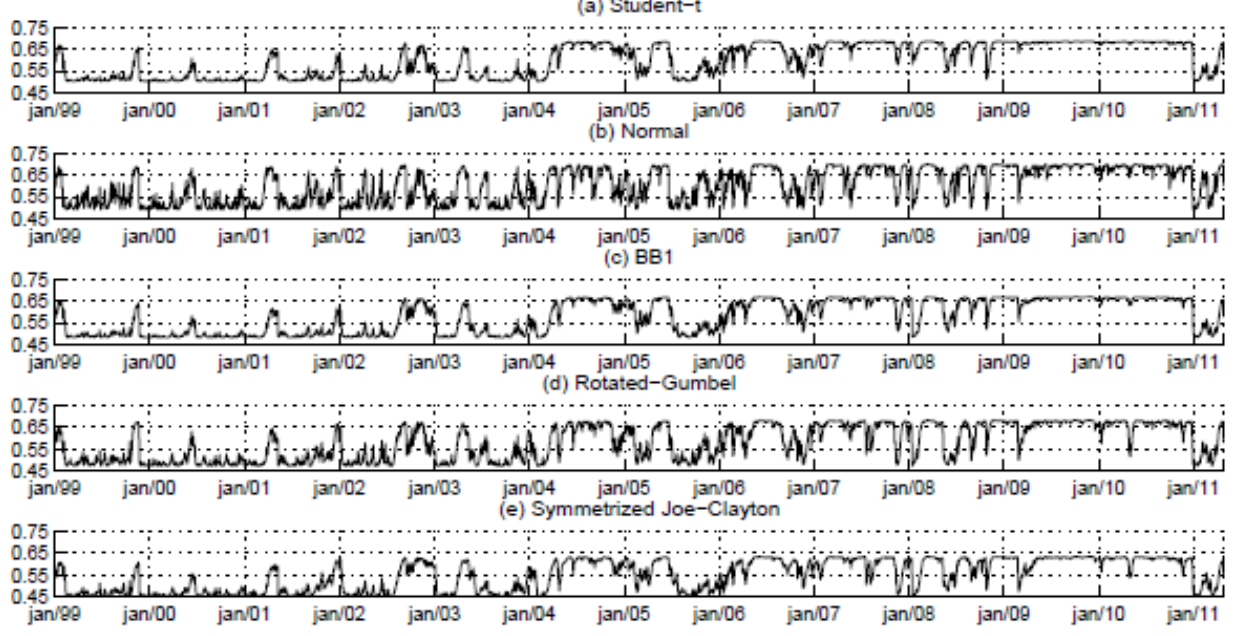

Figure C7. The evolution of the Kendall's tau computed based on the J\&R copulas, for the FTSE-DAX pair

(a) Student- $t$
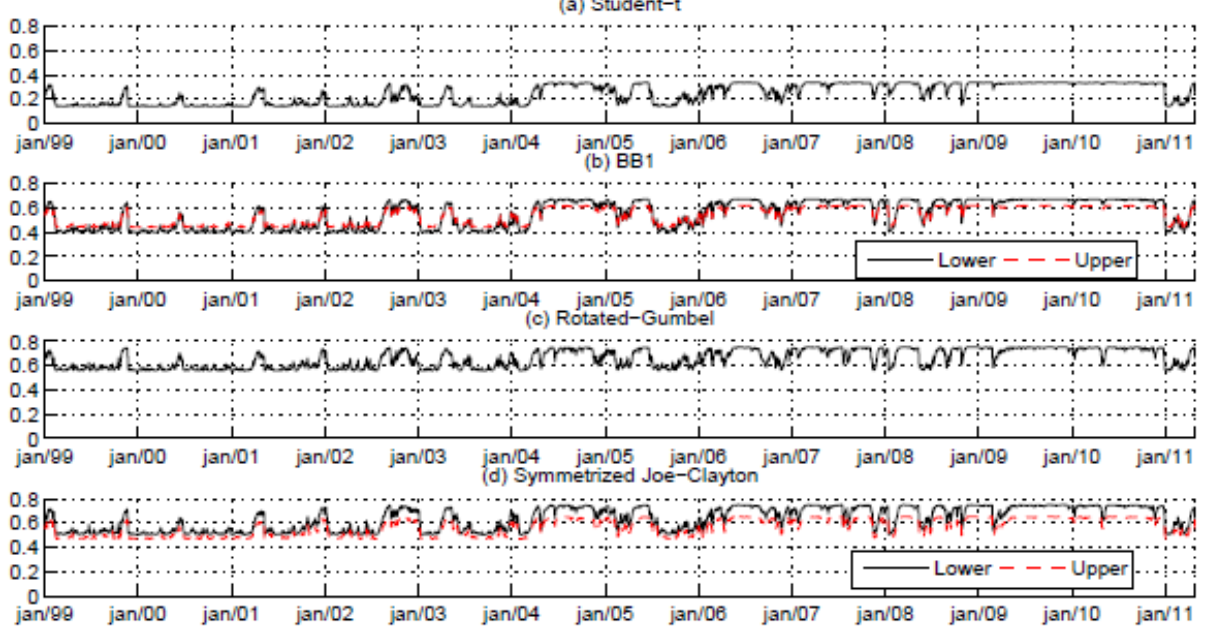

Figure C8. The evolutions of the tail dependence parameters computed based on the J\&R copulas, for the FTSE-DAX pair

(a) Student-t

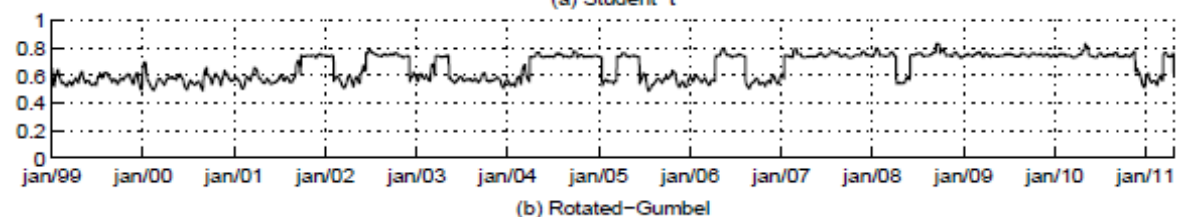

(b) Rotated-Gumbel

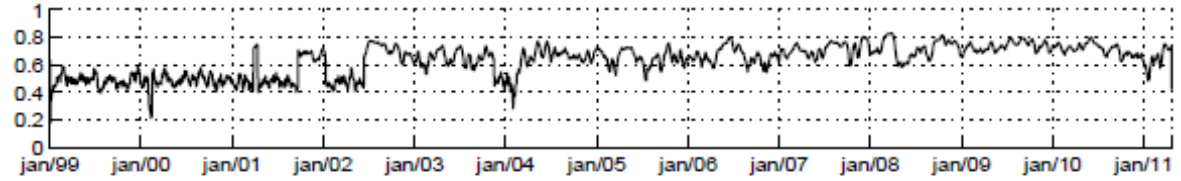

(c) Symmetrized Joe-Clayton

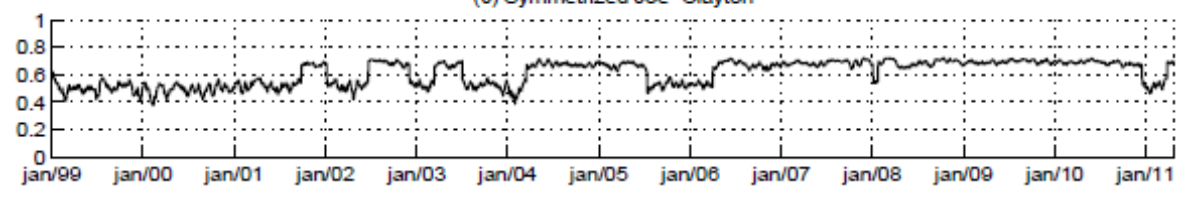

Figure C9. The evolution of the Kendall's tau computed based on the SFZ\&D copulas, for the FTSE-CAC pair 
(a) Student- $t$
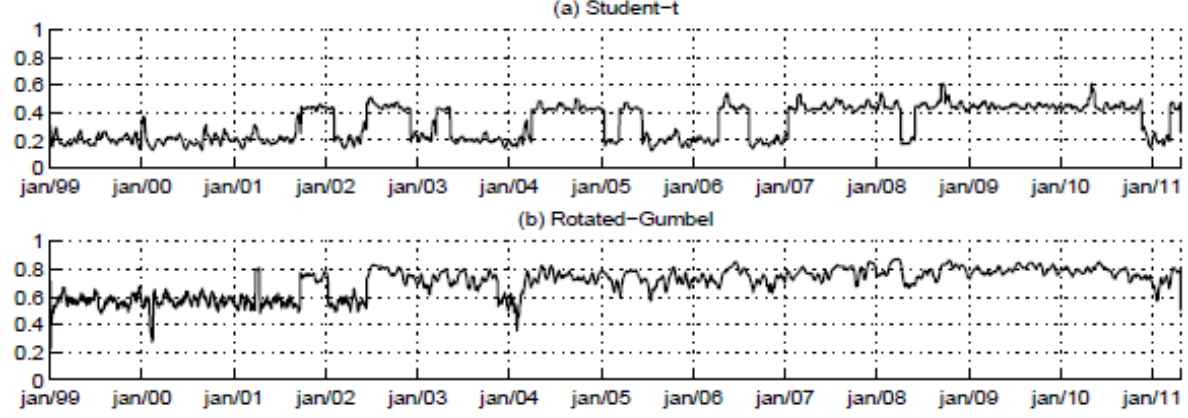

(c) Symmetrized Joe-Clayton

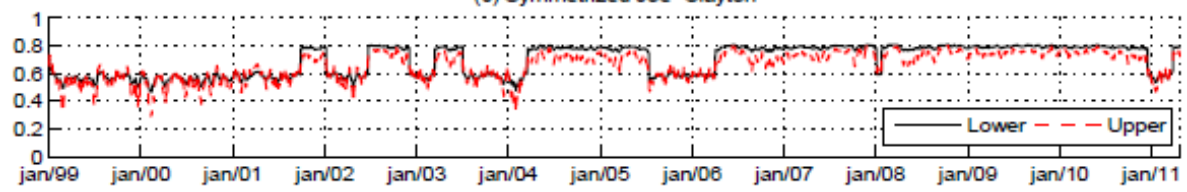

Figure C10. The evolutions of the tail dependence parameters computed based on the SFZ\&D copulas, for the FTSE-CAC pair

(a) Student-t

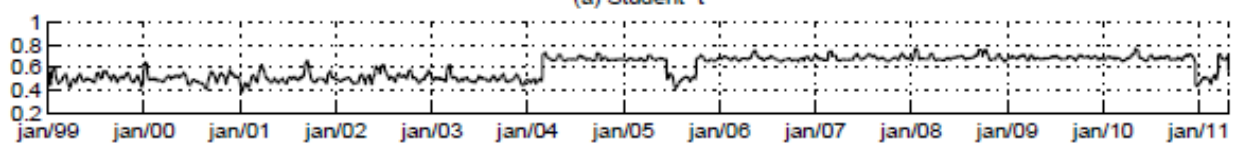

(b) Normal
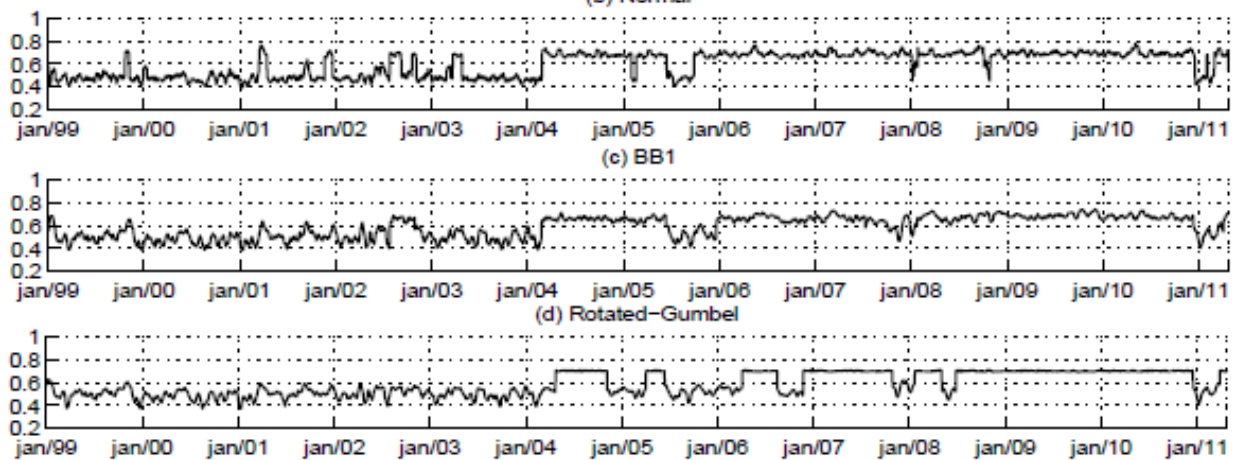

Figure C11. The evolution of the Kendall's tau computed based on the SFZ\&D copulas, for the FTSE-DAX pair

(a) Student-t

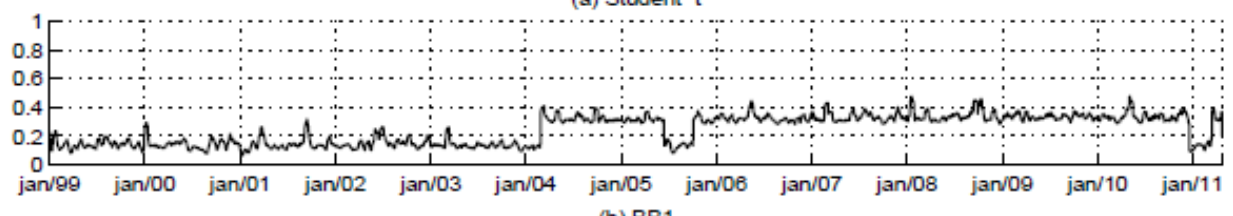

(b) BB1

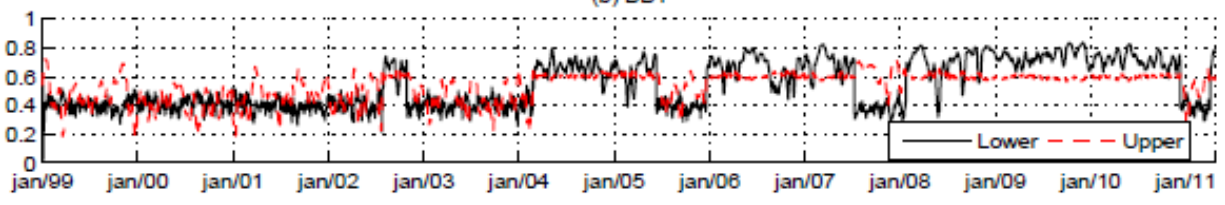

(c) Rotated-Gumbel

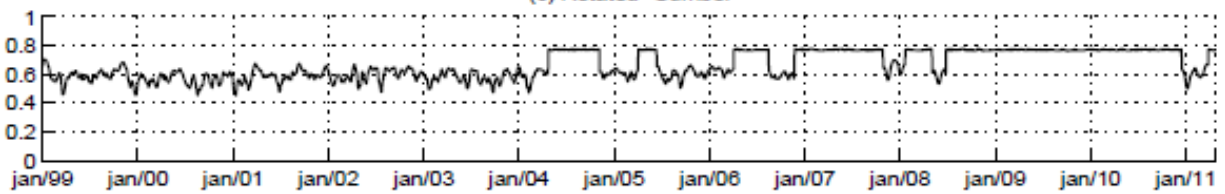

Figure C12. The evolutions of the tail dependence parameters computed based on the SFZ\&D copulas, for the FTSE-DAX pair 
(a) Kendall's tau path

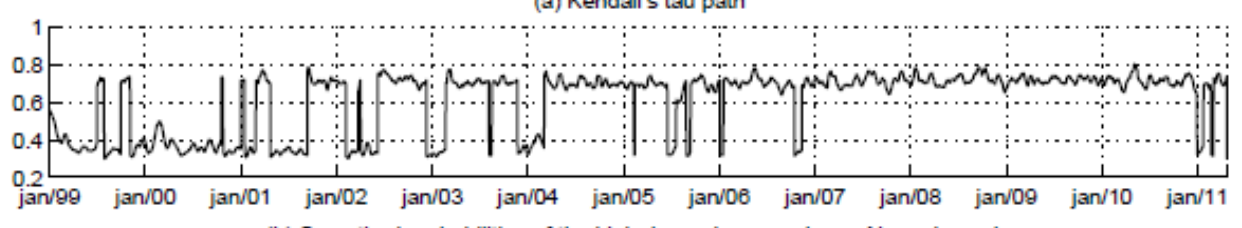

(b) Smoothed probabilities of the high dependence regime - Normal copula

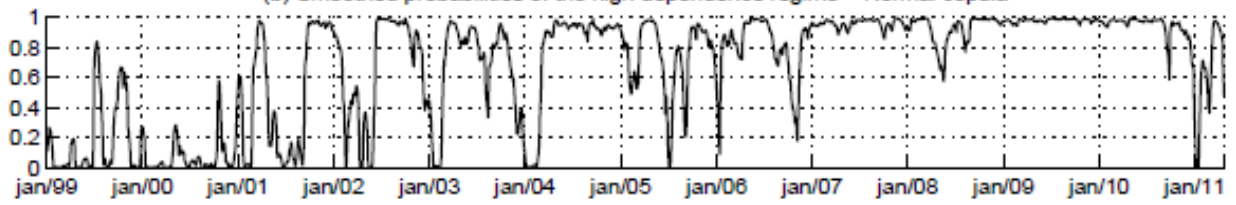

(c) Smoothed probabilities of the low dependence regime - Clayton copula

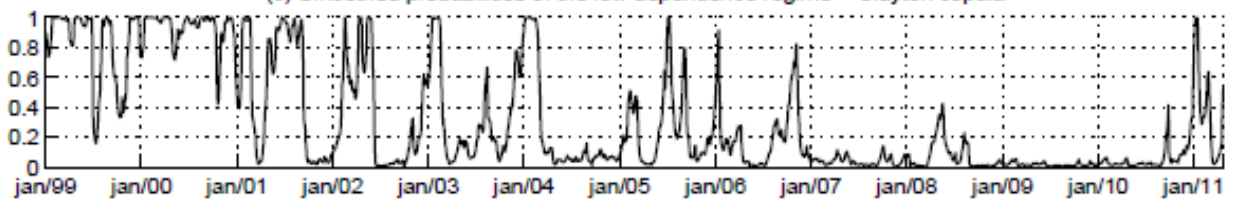

Figure C13. The evolution of the Kendall's tau computed based on the Normal-Clayton Markov switching copula and the smoothed probabilities of the high and low dependence regimes, for the FTSE-CAC pair

\section{Copyrights}

Copyright for this article is retained by the author(s), with first publication rights granted to the journal.

This is an open-access article distributed under the terms and conditions of the Creative Commons Attribution license (http://creativecommons.org/licenses/by/4.0/). 